\title{
Bipolar Soft Sets: Relations between Them and Ordinary Points and Their Applications
}

\author{
Tareq M. Al-shami iD \\ Department of Mathematics, Sana'a University, Sana'a, Yemen \\ Correspondence should be addressed to Tareq M. Al-shami; tareqalshami83@gmail.com
}

Received 2 November 2020; Revised 19 November 2020; Accepted 29 November 2020; Published 29 January 2021

Academic Editor: Ahmed Mostafa Khalil

Copyright (C) 2021 Tareq M. Al-shami. This is an open access article distributed under the Creative Commons Attribution License, which permits unrestricted use, distribution, and reproduction in any medium, provided the original work is properly cited.

\begin{abstract}
Bipolar soft set is formulated by two soft sets; one of them provides us the positive information and the other provides us the negative information. The philosophy of bipolarity is that human judgment is based on two sides, positive and negative, and we choose the one which is stronger. In this paper, we introduce novel belong and nonbelong relations between a bipolar soft set and an ordinary point. These relations are considered as one of the unique characteristics of bipolar soft sets which are somewhat expression of the degrees of membership and nonmembership of an element. We discuss essential properties and derive the sufficient conditions of some equivalence of these relations. We also define the concept of soft mappings between two classes of bipolar soft sets and study the behaviors of an ordinary point under these soft mappings with respect to all relations introduced herein. Then, we apply bipolar soft sets to build an optimal choice application. We give an algorithm of this application and show the method for implementing this algorithm by an illustrative example. In conclusion, it can be noted that the relations defined herein give another viewpoint to explore the concepts of bipolar soft topology, in particular, soft separation axioms and soft covers.
\end{abstract}

\section{Introduction}

Many problems in engineering, artificial intelligence, economy, environmental science, social science, etc. involve data that contain ambiguity/vagueness. Therefore, traditional methods which were based on the exact case may not be convenient for solving or modeling them.

From this point, the need of new theories that help to surpass these types of instabilities arose. With the passage of time, engineers and mathematicians found alternative approaches to solve the problems that contain ambiguity/ vagueness such as probability theory, fuzzy set [1], intuitionistic fuzzy set [2], and rough set [3].

However, all these tools require the prespecification of some parameters to start with, for example, an equivalence relation in rough set theory and density function in probability theory. According to the fuzzy set theory, the difficulties in many problems appear in two sides: the first one is how we can determine a membership function for each particular case, and the second difficulty is the extremely individual characteristic of a membership function. That is, everyone understands the meaning of the membership function equal to 0.85 in his own manner.

To cope with these difficulties, Molodtsov [4] proposed a new approach, namely, soft sets. Simply, soft set is defined as a map of a set of parameters into the power set of the universe of discourse. He demonstrated the efficiency of soft sets in handling complicated problems compared with the probability theory and fuzzy sets theory. Then, many researchers have studied the properties, operations, and applications of soft set theory (see, for example, [5-13]). The authors of $[14,15]$ explained the relationship between soft set, rough set, and fuzzy set.

In recent years, a number of authors have extensively explored some extensions of soft set. These studies go in two directions: The first one is given by generalizing the structure of soft set. This leads to defining double framed soft set [16], bipolar soft set [17], binary soft set [18], and N-soft set [19]. The second one is introduced by combining soft set (or its updated forms) with rough set or fuzzy set or both. This leads 
to defining fuzzy soft set [12], fuzzy bipolar soft set [20], bipolar fuzzy soft set [21], soft rough set [14], bipolar soft rough set [22], and modified rough bipolar soft set [23].

In [24], the authors pointed out that human decisions rely on two types of information having positive and negative flavor. In this case, if we determine a set of parameters which gives us positive data, we also need to know an associated set of oppositely meaning parameters called the "not set of parameters." For instance, if a parameter $a$ stands for a "tall" characteristic, then not $a$ stands for a characteristic of "not tall." It should be noted that a characteristic of "not tall" does not mean "short." That is, the members who are tall and not tall need not be the universal set. Due to the importance of providing positive and negative aspects of data at a time, Shabir and Naz [17] formulated the idea of bipolar soft sets and discussed its application to decisionmaking problems. After this study, bipolar soft sets are gaining momentum among researchers. In 2015, Karaaslan and Karataş [25] redefined a bipolar soft set using a bijective map between a set of parameters and its negative. They also provided a decision-making manner using bipolar soft set with the aid of an example. In [26], the authors revealed some algebraic structures of bipolar soft sets. It was followed by Karaaslan et al. [27] who studied a group structure on bipolar soft sets.

In 2017, Shabir and Bakhtawar [28] first introduced the concept of bipolar soft topological spaces over a crisp set along with an investigation into bipolar soft compactness and connectedness. Then, Öztürk [29] further discussed the concepts of interior and closure operators, basis, and subspace in the bipolar soft topological spaces. Fadel and Hassan [30] presented the concepts of bipolar soft separation axioms and established fundamental properties. Recently, Fadel and Dzul-Kifli [31] have generalized the concept of bipolar soft topological spaces given in [28] by redefining it on a bipolar soft set. They have presented its main notions and described properties along with some illustrative examples.

In 2018, the authors of [32] came up with the idea of partial belong and total nonbelong relations between an ordinary point and soft set which somewhat indicate the degree of membership and nonmembership of an element. In fact, these relations widely open the door to the studying and redefining of many soft topological notions and the obtaining of many fruitful properties. Some applications of these two relations in the domains of soft separation axioms and decision-making problems were introduced in [33-35]. The authors of [36-38] applied these relations to study separation axioms on soft topological ordered spaces and supra soft topological ordered spaces.

The rest of this paper is organized as follows. Section 2 involves some operations and properties of bipolar soft sets. In Section 3, we define five sorts of belong relations between bipolar soft set and ordinary point called positively partial belong, negatively partial belong, partial belong, positively total belong, and negatively total belong relations; we also define six sorts of nonbelong relations between a bipolar soft set and an ordinary point called positively partial nonbelong, negatively partial nonbelong, partial nonbelong, positively total nonbelong, negatively total nonbelong, and total nonbelong relations. Then, we ascertained their behaviors with respect to the operations of soft union and intersection. Later, we define the concept of soft mappings between two classes of weak bipolar soft sets and discuss the relationship between an ordinary point and its image and preimage with respect to the different types of belong and nonbelong relations. In Section 4, we make use of bipolar soft sets to construct an application of optimum choice and present its algorithm. We give a practical example to illustrate how this algorithm can be applied. In the end, we outline the main obtained results and suggest some future work in Section 5.

\section{Preliminaries}

In the following, we recall some definitions related to bipolar soft sets.

Through this paper, $A, B, C, D, E, M, N$ denote the sets of parameters, and $X, Y$ denote the initial universal sets.

Definition 1 (see [4]). A map $f$ of $A$ into the power set of a nonempty set $X$ is called a soft set over $X$, where $X$ is an initial universal set and $A$ is a set of parameters.

A soft set is symbolized by an ordered pair $(f, A)$ and it is expressed as a set of ordered pairs:

$$
(f, A)=\left\{(a, f(a)): a \in A \text { and } f(a) \in 2^{X}\right\} .
$$

Definition 2 (see [17]). A bipolar soft set is a triple $(f, g, A)$ over a nonempty set $X$ with a set of parameters $A$, where $f: A \longrightarrow 2^{X}$ and $g: \neg A \longrightarrow 2^{X}$ are two crisp maps such that $f(a) \cap g(\neg a)=\varnothing$ for each $a \in A$.

A bipolar soft set is expressed as a set of ordered triples:

$$
(f, g, A)=\left\{(a, f(a), g(\neg a)): a \in A \text { and } f(a), g(\neg a) \in 2^{X}\right\} \text {. }
$$

A class of all bipolar soft sets defined over $X$ with all sets of parameters which are subsets of $A$ is symbolized by $C\left(X_{A}\right)$.

Henceforth, we consider that any bipolar soft set is defined on $X \neq \varnothing$, unless otherwise specified.

Example 1. Let $X=\left\{x_{1}, x_{2}, \ldots, x_{6}\right\}$ be the universe containing set six cars and $A=\left\{a_{1}, a_{2}, a_{3}, a_{4}\right\}$ be a set of parameters, where $a_{1}, a_{2}, a_{3}$, and $a_{4}$ stand for "expensive," "in good repair," "red colored," and "made in Japan," respectively.

Let $f: A \longrightarrow 2^{X}$ and $g: \neg A \longrightarrow 2^{X}$ be two maps given as follows:

$$
\begin{aligned}
& f\left(a_{1}\right)=\left\{x_{3}, x_{4}\right\}, g\left(\neg a_{1}\right)=\left\{x_{1}\right\}, \\
& f\left(a_{2}\right)=\left\{x_{3}, x_{5}, x_{6}\right\}, \quad g\left(\neg a_{2}\right)=\varnothing, \\
& f\left(a_{3}\right)=\left\{x_{1}, x_{2}, x_{4}\right\}, \quad g\left(\neg a_{3}\right)=\left\{x_{3}, x_{5}, x_{6}\right\}, \\
& f\left(a_{4}\right)=\left\{x_{3}, x_{6}\right\}, \quad g\left(\neg a_{4}\right)=\left\{x_{1}, x_{2}, x_{4}, x_{5}\right\} .
\end{aligned}
$$

Now, we can describe this system using a bipolar soft set as follows: 


$$
\begin{aligned}
(f, g, A)= & \left\{\left(a_{1},\left\{x_{3}, x_{4}\right\},\left\{x_{3}, x_{1}\right\}\right),\left(a_{2},\left\{x_{3}, x_{5}, x_{6}\right\}, \varnothing\right),\right. \\
& \left(a_{3},\left\{x_{1}, x_{2}, x_{4}\right\},\left\{x_{3}, x_{5}, x_{6}\right\}\right),\left(a_{4},\left\{x_{3}, x_{6}\right\},\right. \\
& \left.\left.\left\{x_{1}, x_{2}, x_{4}, x_{5}\right\}\right)\right\} .
\end{aligned}
$$

Definition 3 (see [17]). A bipolar soft set $(f, g, A)$ is said to be

(i) A relative null bipolar soft set if $f(a)$ equals the empty set and $g(\neg a)$ equals the universal set for each $a \in A$. It is symbolized by $(\Phi, X, A)$.

(ii) A relative absolute bipolar soft set if $f(a)$ equals the universal set and $g(\neg a)$ equals the empty set for each $a \in A$. It is symbolized by $(X, \Phi, A)$.

Definition 4 (see [17]). The intersection of two bipolar soft sets $\left(f_{1}, f_{2}, A\right)$ and $\left(g_{1}, g_{2}, B\right)$ is a bipolar soft set $\left(h_{1}, h_{2}, C\right)$ such that $C=A \cap B \neq \varnothing$ and the two mappings $h_{1}: C \longrightarrow 2^{X}$ and $h_{2}: \neg C \longrightarrow 2^{X}$ are given by

$$
\begin{aligned}
h_{1}(c) & =f_{1}(c) \cap g_{1}(c), \\
h_{2}(\neg c) & =f_{2}(\neg c) \cup g_{2}(\neg c) .
\end{aligned}
$$

It is symbolized by $\left(f_{1}, f_{2}, A\right) \widetilde{\cap}\left(g_{1}, g_{2}, B\right)$.

Definition 5 (see [17]). The union of two bipolar soft sets $\left(f_{1}, f_{2}, A\right)$ and $\left(g_{1}, g_{2}, B\right)$ is a bipolar soft set $\left(h_{1}, h_{2}, C\right)$, where $C=A \cup B$ and the two mappings $h_{1}: C \longrightarrow 2^{X}$ and $h_{2}: \neg C \longrightarrow 2^{X}$ are given by

$$
\begin{aligned}
h_{1}(c) & = \begin{cases}f_{1}(c): & c \in A \backslash B, \\
g_{1}(c): & c \in B \backslash A, \\
f_{1}(c) \cup g_{1}(c): & c \in A \cap B,\end{cases} \\
h_{2}(\neg c) & = \begin{cases}f_{2}(\neg c): & \neg c \in \neg A \backslash \neg B, \\
g_{2}(\neg c): & \neg c \in \neg B \backslash \neg A, \\
f_{2}(\neg c) \cap g_{2}(\neg c): & \neg c \in \neg A \cap \neg B .\end{cases}
\end{aligned}
$$

It is symbolized by $\left(f_{1}, g_{1}, A\right) \widetilde{U}\left(f_{2}, g_{2}, B\right)$.

Definition 6 (see [17]). A bipolar soft set $(f, g, A)$ is said to be a subset of a bipolar soft set $(h, l, B)$, denoted by $(f, g, A) \tilde{\subseteq}(h, l, B)$, provided that

(i) $A \subseteq B$

(ii) For all $a \in A$, we have $f(a) \subseteq h(a)$ and $l(\neg a) \subseteq g(\neg a)$

The bipolar soft sets $(f, g, A)$ and $(h, l, B)$ are said to be soft equal if $(f, g, A) \tilde{\simeq}(h, l, B)$ and $(h, l, B) \widetilde{\simeq}(f, g, A)$.

Definition 7 (see [17]). The relative complement of a bipolar soft set $(f, g, A)$ is a bipolar soft set $(f, g, A)^{\prime}=\left(f^{\prime}, g^{\prime}, A\right)$, where $f^{\prime}: A \longrightarrow 2^{X}$ and $g^{\prime}: \neg A \longrightarrow 2^{X}$ are defined as follows:

$$
\begin{aligned}
f^{\prime}(a) & =g(\neg a), \\
g^{\prime}(\neg a) & =f(a),
\end{aligned}
$$

for all $a \in A$ and $\neg a \in \neg A$.

\section{Belong and Nonbelong Relations between Bipolar Soft Sets and Ordinary Points}

In this section, we initiate five types of memberships and six types of nonmemberships between bipolar soft set and ordinary point and ascertain the relationships between them. We investigate their main properties in terms of soft union and intersection operators, the product of bipolar soft sets and soft mappings.

Definition 8. Let $(f, g, A)$ be a bipolar soft set and $x \in X$. We say that

(i) $x \Subset_{\mathrm{pp}}(f, g, A)$, reading as $x$ positively partially belongs to $(f, g, A)$, if $x \in f(a)$ for some $a \in A$

(ii) $x \Subset_{\mathrm{np}}(f, g, A)$, reading as $x$ negatively partially belongs to $(f, g, A)$, if $x \in g(\neg a)$ for some $\neg a \in \neg A$

(iii) $x \Subset_{p}(f, g, A)$, reading as $x$ partially belongs to $(f, g, A)$, if $x \in f(a)$ and $x \in g\left(\neg a^{\prime}\right)$ for some $a \in A$ and $\neg a^{\prime} \in \neg A$

(iv) $x \epsilon_{\mathrm{pt}}(f, g, A)$, reading as $x$ positively totally belongs to $(f, g, A)$, if $x \in f(a)$ for all $a \in A$

(v) $x \epsilon_{\mathrm{nt}}(f, g, A)$, reading as $x$ negatively totally belongs to $(f, g, A)$, if $x \in g(\neg a)$ for all $\neg a \in \neg A$

Definition 9. Let $(f, g, A)$ be a bipolar soft set and $x \in X$. We say that

(i) $x \notin_{\mathrm{pp}}(f, g, A)$, reading as $x$ does not positively partially belong to $(f, g, A)$, if $x \notin f(a)$ for some $a \in A$

(ii) $x \notin_{\mathrm{np}}(f, g, A)$, reading as $x$ does not negatively partially belong to $(f, g, A)$, if $x \notin g(\neg a)$ for some $\neg a \in \neg A$

(iii) $x \notin_{p}(f, g, A)$, reading as $x$ does not partially belong to $(f, g, A)$, if $x \notin f(a)$ and $x \notin g\left(\neg a^{\prime}\right)$ for some $a \in A$ and $\neg a^{\prime} \in \neg A$

(iv) $x \notin{ }_{\mathrm{pt}}(f, g, A)$, reading as $x$ does not positively totally belong to $(f, g, A)$, if $x \notin f(a)$ for all $a \in A$.

(v) $x \notin{ }_{\mathrm{nt}}(f, g, A)$, reading as $x$ does not negatively totally belong to $(f, g, A)$, if $x \notin g(\neg a)$ for all $\neg a \in \neg A$

(vi) $x \notin_{t}(f, g, A)$, reading as $x$ does not totally belong to $(f, g, A)$, if $x \notin f(a)$ and $x \notin g(\neg a)$ for all $a \in A$ and $\neg a \in \neg A$

To well understand the results initiated in this work, we give the following example and remark.

Example 2. Let $A=\left\{a_{1}, a_{2}, a_{3}\right\}$ be a set of parameters and $(f, g, A)$ be bipolar soft set over $X=\left\{x_{1}, x_{2}, \ldots, x_{10}\right\}$ defined as follows: 


$$
(f, g, A)=\left\{\left(a_{1},\left\{x_{1}\right\},\left\{x_{2}, x_{4}, x_{10}\right\}\right),\left(a_{2}, \varnothing,\left\{x_{4}, x_{10}\right\}\right),\left(a_{3},\left\{x_{2}, x_{3}\right\},\left\{x_{4}, x_{5}, x_{10}\right\}\right)\right\} .
$$

Then, it follows from Definition 7 that $\left(f^{\prime}, g^{\prime}, A\right)=(g, f, A)$. We note the following:

$$
(f, g, A) \widetilde{\cap}\left(f^{\prime}, g^{\prime}, A\right)=\left\{\left(a_{1}, \varnothing,\left\{x_{1}, x_{2}, x_{4}, x_{10}\right\}\right),\left(a_{2}, \varnothing,\left\{x_{4}, x_{10}\right\}\right),\left(a_{3}, \varnothing,\left\{x_{2}, x_{3}, x_{4}, x_{5}, x_{10}\right\}\right)\right\} \neq(\Phi, X, A)
$$

That is, the intersection of a bipolar soft set and its relative complement need not be the null bipolar soft set.

$$
(f, g, A) \widetilde{\cup}\left(f^{\prime}, g^{\prime}, A\right)=\left\{\left(a_{1},\left\{x_{1}, x_{2}, x_{4}, x_{10}\right\}, \varnothing\right),\left(a_{2},\left\{x_{4}, x_{10}\right\}, \varnothing\right),\left(a_{3},\left\{x_{2}, x_{3}, x_{4}, x_{5}, x_{10}\right\}, \varnothing\right)\right\} \neq(X, \Phi, A)
$$

That is, the union of a bipolar soft set and its relative complement need not be the absolute bipolar soft set.

Remark 1. The possibility of existence and nonexistence of an element in the same place is well-known in the quantum physics, that is, corresponding of a thing and its opposite. This matter also occurs here with respect to positively partial belong and positively partial nonbelong relations; negatively partial belong and negatively partial nonbelong relations; and partial belong and partial nonbelong relations. For instance, in Example 2, it can be seen that

$$
\begin{aligned}
& x_{1} \notin_{\mathrm{pp}}(f, g, A), \\
& x_{1} \Subset_{\mathrm{pp}}(f, g, A), \\
& x_{2} \notin_{\mathrm{np}}(f, g, A), \\
& x_{2} \Subset_{\mathrm{np}}(f, g, A), \\
& x_{2} \notin_{p}(f, g, A), \\
& x_{2} \Subset_{p}(f, g, A) .
\end{aligned}
$$

Proposition 1. For two bipolar soft sets $(f, g, A)$ and $(h, l, A)$ and $x \in X$, we have the following results:
(i) $x \Subset_{p p}(f, g, A) \Longleftrightarrow x \Subset_{n p}\left(f^{\prime}, g^{\prime}, A\right)$.
(ii) $x \Subset_{p}(f, g, A) \Longleftrightarrow x \Subset_{p}\left(f^{\prime}, g^{\prime}, A\right)$.
(iii) $x \epsilon_{p t}(f, g, A) \Longleftrightarrow x \epsilon_{n t}\left(f^{\prime}, g^{\prime}, A\right)$.

Proof

(i) $x \Subset_{\mathrm{pp}}(f, g, A) \Leftrightarrow x \in f(a)$ for some $a \in A \Leftrightarrow x \in g^{\prime}$ $(\neg a)=f(a)$ for some $\neg a \in \neg A \Leftrightarrow x \Subset_{\mathrm{np}}\left(f^{\prime}, g^{\prime}, A\right)$.

(ii) $x \Subset_{p}(f, g, A) \Leftrightarrow x \in f(a)$ and $x \in g(\neg a)$ for some $a \in A$ and $\neg a \in \neg A \Leftrightarrow x \in f^{\prime}(a)=g(\neg a)$ and $x \in g^{\prime}(\neg a)=f(a)$ for some $a \in A$ and $\neg a \in \neg A \Leftrightarrow x \Subset_{p}\left(f^{\prime}, g^{\prime}, A\right)$.

(iii) $x \in \epsilon_{\mathrm{pt}}(f, g, A) \Leftrightarrow x \in f(a)$ for all $a \in A \Leftrightarrow x \in g^{\prime}$ $(\neg a)=f(a)$ for all $\neg a \in \neg A \Leftrightarrow x \in_{\mathrm{nt}}\left(f^{\prime}, g^{\prime}, A\right)$.
The proofs of the following results follow from Definition 8 .

Proposition 2. For two bipolar soft sets $(f, g, A)$ and $(h, l, A)$ and $x \in X$, we have the following results:

(i) $x \Subset_{p}(f, g, A) \Leftrightarrow x \Subset_{p p}(f, g, A)$ and $x \Subset_{n p}(f, g, A)$

(ii) $x \epsilon_{p t}(f, g, A) \Leftrightarrow x \Subset_{p p}(f, g, A)$

(iii) $x \epsilon_{n t}(f, g, A) \Leftrightarrow x \Subset_{n p}(f, g, A)$

Proposition 3. For two bipolar soft sets $(f, g, A)$ and $(h, l, A)$ and $x \in X$, we have the following results:

(i) $x \notin_{p}(f, g, A) \Leftrightarrow x \notin_{p p}(f, g, A)$ and $x \notin_{n p}(f, g, A)$

(ii) $x \notin_{p}(f, g, A) \Leftrightarrow x \notin_{p p}(f, g, A)$ and $x \notin_{n p}(f, g, A)$

(iii) $x \notin_{p t}(f, g, A) \Leftrightarrow x \notin_{p p}(f, g, A)$

(iv) $x \notin_{n t}(f, g, A) \Leftrightarrow x \notin_{n p}(f, g, A)$

(v) $x \notin_{t}(f, g, A) \Leftrightarrow x \notin_{p}(f, g, A)$

To show that the converse of (ii) and (iii) of Proposition 2 and (ii) to ( $v$ ) of Proposition 3 fails, we give the following example.

Example 3. In Example 2, we note the following:

(i) $x_{1} \Subset_{\mathrm{pp}}(f, g, A)$ and $x_{5} \Subset_{\mathrm{np}}(f, g, A)$, but $x_{1} \in_{p t}$ $(f, g, A)$ and $x_{5} \in_{\mathrm{nt}}(f, g, A)$ do not hold true

(ii) $x_{2} \notin_{p}(f, g, A), x_{2} \notin_{\mathrm{pp}}(f, g, A)$ and $x_{2} \notin_{\mathrm{np}}(f, g, A)$, but $\quad x_{2} \notin_{t}(f, g, A), \quad x_{2} \notin_{\mathrm{pt}}(f, g, A), \quad$ and $x_{2} \notin \mathrm{nt}(f, g, A)$ do not hold true

Proposition 4. Let $(f, g, A)$ and $(h, l, A)$ be bipolar soft sets such that $(f, g, A) \widetilde{\simeq}(h, l, A)$. Then, we have the following results:

(i) if $x \Subset_{p p}(f, g, A) \quad$ (resp., $\quad x \epsilon_{p t}(f, g, A)$ ), then $x \Subset_{p p}(h, l, A)$ (resp., $\left.x \epsilon_{p t}(h, l, A)\right)$

(ii) if $x \in_{n t}(h, l, A)$, then $x \epsilon_{n t}(f, g, A)$

(iii) if $\quad x \notin_{p p}(h, l, A) \quad$ (resp., $\left.\quad x \notin_{p t}(h, l, A)\right)$, then $x \Subset_{p p}(f, g, A)($ resp., $x \notin p t(f, g, A))$ 
(iv) if $x \notin_{n t}(f, g, A)$, then $x \notin{ }_{n t}(h, l, A)$

Proof. The proof is straightforward.

Proposition 5. For two bipolar soft sets $(f, g, A)$ and $(h, l, A)$ and $x \in X$, we have the following results:

(i) $x \Subset_{p p}(f, g, A)$ or $x \Subset_{p p}(h, l, A) \Leftrightarrow x \Subset_{p p}(f, g, A) \widetilde{U}$ $(h, l, A)$

(ii) $x \Subset_{n p}(f, g, A) \widetilde{\cup}(h, l, A) \Rightarrow x \Subset_{n p}(f, g, A)$ and $x \Subset_{n p}$ $(h, l, A)$

(iii) $x \Subset_{p}(f, g, A) \widetilde{\cup}(h, l, A) \Rightarrow x \Subset_{p}(f, g, A)$ or $x \Subset_{p}(h$, $l, A)$

(iv) $x \epsilon_{p t}(f, g, A) \quad$ or $\quad x \epsilon_{p t}(h, l, A) \Rightarrow x \epsilon_{p t}(f, g, A) \widetilde{U}$ $(h, l, A)$

(v) $x \epsilon_{n t}(f, g, A)$ and $x \epsilon_{n t}(h, l, A) \Rightarrow x \epsilon_{n t}(f, g, A) \widetilde{U}$ $(h, l, A)$

(vi) $x \Subset_{p p}(f, g, A) \widetilde{\cap}(h, l, A) \Rightarrow x \Subset_{p p}(f, g, A)$ and $x \Subset_{p p}$ $(h, l, A)$

(vii) $x \Subset_{n p}(f, g, A) \widetilde{\cap}(h, l, A) \Rightarrow x \Subset_{n p}(f, g, A)$ or $x \Subset_{n p}$ $(h, l, A)$

(viii) $x \Subset_{p}(f, g, A) \widetilde{\cap}(h, l, A) \Rightarrow x \Subset_{p}(f, g, A)$ or $\quad x \Subset_{p}$ $(h, l, A)$

(ix) $x \epsilon_{p t}(f, g, A)$ and $x \epsilon_{p t}(h, l, A) \Rightarrow x \epsilon_{p t}(f, g, A) \widetilde{\cap}$ $(h, l, A)$

(x) $x \epsilon_{n t}(f, g, A)$ and $x \epsilon_{n t}(h, l, A) \Rightarrow x \epsilon_{n t}(f, g, A) \widetilde{\cap}$ $(h, l, A)$

Proof. We will just prove (i), (iv), (v), (vi), and (ix).

Since $(f, g, A)$ and $(h, l, A)$ are subsets of $(f, g, A) \widetilde{\cup}(h, l, A)$, then the necessary parts of (i), (iv), and (vi) hold; since $(f, g, A) \widetilde{\cap}(h, l, A)$ are subsets of $(f, g, A)$ and $(h, l, A)$, then the sufficient parts of (v) and (ix) hold.

To prove the sufficient part of (i), let $x \Subset_{\mathrm{pp}}(f, g, A) \widetilde{\cup}(h, l, A)$. Then, $x \in f(a) \cup h(a)$ for some $a \in A$. Therefore, $x \in f(a)$ or $h(a)$ for some $a \in A$, and hence $x \Subset_{\mathrm{pp}}(f, g, A)$ or $x \Subset_{\mathrm{pp}}(h, l, A)$.

To prove the necessary part of $(\mathrm{v})$, let $x \in_{\mathrm{nt}}(f, g, A)$ and $x \in_{\mathrm{nt}}(h, l, A)$. Then, for all $\neg a \in \neg A$, we have $x \in g(\neg a)$ and $x \in l(\neg a)$. Therefore, $x \in g(\neg a) \cap l(\neg a)$ for all $\neg a \in \neg A$. Hence, $x \in_{\mathrm{nt}}(f, g, A) \widetilde{\cup}(h, l, A)$.

Similarly, one can prove the necessary part of (ix).

We provide the next example to clarify that the converse of results (ii), (iii), (vi), and (viii) to (x) of Proposition 5 fails.

Example 4. Let $A=\left\{a_{1}, a_{2}\right\}$ be a set of parameters and $(f, g, A),(h, l, A)$ be bipolar soft sets over $X=\left\{x_{1}, x_{2}\right.$, $\left.x_{3}, x_{4}, x_{5}, x_{6}, x_{7}\right\}$ defined as follows:

$$
\begin{aligned}
(f, g, A) & =\left\{\left(a_{1},\left\{x_{1}, x_{3}\right\},\left\{x_{5}, x_{7}\right\}\right),\left(a_{2},\left\{x_{4}, x_{6}\right\},\left\{x_{3}, x_{5}\right\}\right)\right\}, \\
(h, l, A) & =\left\{\left(a_{1},\left\{x_{1}, x_{4}\right\},\left\{x_{3}, x_{4}, x_{5}\right\}\right),\left(a_{2},\left\{x_{2}\right\},\left\{x_{1}, x_{3}, x_{5}, x_{7}\right\}\right)\right\} .
\end{aligned}
$$

Then, $(f, g, A) \widetilde{\cup}(h, l, A)=\left\{\left(a_{1},\left\{x_{1}, x_{3}, x_{4}\right\},\left\{x_{5}\right\}\right),\left(a_{2}\right.\right.$, $\left.\left.\left\{x_{2}, x_{4}, x_{6}\right\},\left\{x_{3}, x_{5}\right\}\right)\right\}$, and $(f, g, A) \cap(h, l, A)=\left\{\left(a_{1},\left\{x_{1}\right\}\right.\right.$, $\left.\left.\left\{x_{3}, x_{4}, x_{5}, x_{7}\right\}\right),\left(a_{2}, \varnothing,\left\{x_{1}, x_{3}, x_{5}, x_{7}\right\}\right)\right\}$
We note the following:

(i) $x_{7} \Subset_{\mathrm{np}}(f, g, A)$ and $x_{7} \Subset_{\mathrm{np}}(h, l, A) x_{7} \Subset_{\mathrm{np}}(h, l, A)$, but $x_{7} \Subset_{n p}(f, g, A) \widetilde{\cup}(h, l, A)$ does not hold true

(ii) $x_{7} \Subset_{p}(f, g, A)$ and $x_{7} \Subset_{p}(h, l, A) x_{7} \Subset_{p}(h, l, A)$, but $x_{7} \Subset_{p}(f, g, A) \widetilde{\cup}(h, l, A)$ and $x_{7} \Subset_{p}(f, g, A) \widetilde{\cap}($ $h, l, A)$ do not hold true

(iii) $x_{4} \Subset_{\mathrm{pt}}(f, g, A) \widetilde{\cup}(h, l, A) x_{4} \Subset_{\mathrm{pt}}(f, g, A) \widetilde{\cup}(h, l, A)$, but $x_{4} \Subset_{\mathrm{pt}}(f, g, A)$ or $x_{4} \Subset_{\mathrm{pt}}(h, l, A)$ does not hold true

(iv) $x_{4} \Subset_{\mathrm{pp}}(f, g, A)$ and $x_{4} \Subset_{\mathrm{pp}}(h, l, A) x_{4} \Subset_{\mathrm{pp}}(h, l, A)$, but $x_{4} \Subset_{\mathrm{pp}}(f, g, A) \widetilde{\cap}(h, l, A)$ does not hold true

(v) $x_{7} \Subset_{\mathrm{nt}}(f, g, A) \widetilde{\cap}(h, l, A) x_{7} \Subset_{\mathrm{nt}}(f, g, A) \widetilde{\cap}(h, l, A)$, but $x_{7} \Subset_{\mathrm{nt}}(f, g, A)$ and $x_{4} \Subset_{\mathrm{pt}}(h, l, A)$ do not hold true

One can prove the following result similarly.

Proposition 6. For two bipolar soft sets $(f, g, A)$ and $(h, l, A)$ over $X$ and $x \in X$, we have the following results:

(i) $x \notin_{p p}(f, g, A) \widetilde{\cup}(h, l, A) \Rightarrow x \notin_{p p}(f, g, A)$ and $x$ $\notin_{p p}(h, l, A)$

(ii) $x \notin_{n p}(f, g, A) \widetilde{\cup}(h, l, A) \Leftrightarrow x \notin_{n p}(f, g, A)$ and $x$ $\notin_{n p}(h, l, A)$

(iii) $x \notin_{p}(f, g, A) \widetilde{\cup}(h, l, A) \Rightarrow x \Subset_{p}(f, g, A)$ and $x$ $\notin_{p}(h, l, A)$

(iv) $x \notin_{p t}(f, g, A) \widetilde{\cup}(h, l, A) \Leftrightarrow x \notin_{p t}(f, g, A)$ and $x$ $\notin_{p t}(h, l, A)$

(v) $x \notin_{n t}(f, g, A) \quad$ or $\quad x \notin_{n t}(h, l, A) \Rightarrow x \quad \notin_{n t}$ $(f, g, A) \cup(h, l, A)$.

(vi) $x \notin_{p p}(f, g, A)$ or $x \notin_{p p}(h, l, A) \Rightarrow x \Subset_{p p}(f, g, A) \widetilde{\cap}$ $(h, l, A)$.

(vii) $x \notin_{n p}(f, g, A) \widetilde{\cap}(h, l, A) \Rightarrow x \notin_{n p}(f, g, A) \quad$ or $x \notin_{n p}(h, l, A)$

(viii) $x \notin_{p}(f, g, A) \widetilde{\cap}(h, l, A) \Rightarrow x \notin_{p}(f, g, A) \quad$ or $\quad x \notin_{p}$ $(h, l, A)$

(ix) $x \notin_{p t}(f, g, A)$ or $x \notin_{p t}(h, l, A) \Rightarrow x \notin_{p t}(f, g, A)$ $\widetilde{\cap}(h, l, A)$.

(x) $x \notin_{n t}(f, g, A) \widetilde{\cap}(h, l, A) \Leftrightarrow x \notin_{n t}(f, g, A)$ and $x$ $\notin_{n t}(h, l, A)$

(xi) $x \notin_{t}(f, g, A) ; \quad x \notin_{t}(h, l, A) \Leftrightarrow x \quad \notin_{t}(f, g, A) \widetilde{\cap}$ $(h, l, A)$; and $x \notin_{t}(f, g, A) \cup(h, l, A)$

Definition 10. A bipolar soft set $(f, g, A)$ is said to be stable if there are two disjoint subsets $U, V$ of $X$ such that $f(a)=U$ for each $a \in A$ and $g(\neg a)=V$ and for each $\neg a \in \neg A$.

$(f, g, A)$ is said to be positive stable if $V=\varnothing$; and $(f, g, A)$ is said to be negative stable if $U=\varnothing$.

It is clear that positive (negative) stable bipolar soft set is stable; however, the converse is not true.

Proposition 7. Let $(f, g, A)$ be a stable bipolar soft set. Then,

(i) $x \Subset_{p p}(f, g, A) \Leftrightarrow x \Subset_{p t}(f, g, A)$

(ii) $x \Subset_{n p}(f, g, A) \Leftrightarrow x \Subset_{n t}(f, g, A)$ 
(iii) $x \notin_{p}(f, g, A) \Leftrightarrow x \notin_{t}(f, g, A)$

(iv) $x \notin_{p p}(f, g, A) \Leftrightarrow x \notin_{p t}(f, g, A)$

(v) $x \notin_{n p}(f, g, A) \Leftrightarrow x \notin_{n t}(f, g, A)$

Proof. Since $(f, g, A)$ is a stable bipolar soft set, then there are two disjoint subsets $U, V$ of $X$ such that $f(a)=U$ for each $a \in A$ and $g(\neg a)=V$ for each $\neg a \in \neg A$. Therefore, the following properties hold:

(i) $x \in f(a)$ for some $a \in A$ if and only if $x \in f(a)$ for each $a \in A$

(ii) $x \in g(\neg a)$ for some $\neg a \in \neg A$ if and only if $x \in g(\neg a)$ for each $\neg a \in \neg A$

(iii) $x \notin f(a)$ for some $a \in A$ and $x \notin g(\neg a)$ for some $\neg a \in \neg A$ if and only if $x \notin f(a)$ for each $a \in A$ and $x \notin g(\neg a)$ for each $\neg a \in \neg A$

(iv) $x \notin f(a)$ for some $a \in A$ if and only if $x \notin f(a)$ for each $a \in A$

(v) $x \notin g(\neg a)$ for some $\neg a \in \neg A$ if and only if $x \notin g(\neg a)$ for each $\neg a \in \neg A$

Hence, the desired results are proved.

Corollary 1. Let $(f, g, A)$ be a positive stable bipolar soft set. Then,

(i) $x \Subset_{p p}(f, g, A) \Leftrightarrow x \Subset_{p t}(f, g, A)$

(ii) $x \notin_{p p}(f, g, A) \Leftrightarrow x \notin_{p t}(f, g, A)$

$\Leftrightarrow x \notin_{p}(f, g, A) \Leftrightarrow x \notin_{t}(f, g, A)$

Corollary 2. Let $(f, g, A)$ be a negative stable bipolar soft set. Then,

(i) $x \Subset_{n p}(f, g, A) \Leftrightarrow x \Subset_{n t}(f, g, A)$

(ii) $x \notin_{n p}(f, g, A) \Leftrightarrow x \notin_{n t}(f, g, A) \Leftrightarrow x \notin_{p}$

$(f, g, A) \Leftrightarrow x \notin_{t}(f, g, A)$

Definition 11. The Cartesian product of two bipolar soft sets $(f, g, A)$ and $(h, l, B)$, denoted by $(f \times h, g \times l, A \times B)$, is defined as $(f \times h)(a, b)=f(a) \times h(b)$ for each $(a, b) \in A \times$ $B$ and $(g \times l)(\neg a, \neg b)=g(\neg a) \times l(\neg b)$ for each $(\neg a, \neg b) \in \neg A \times \neg B$.

Proposition 8. We have the next four results:

(i) $(x, y) \Subset_{p p}(f, g, A) \times(h, l, B)$ if and only if $x \Subset_{p p}(f, g, A)$ and $y \Subset_{p p}(h, l, B)$

(ii) $(x, y) \Subset_{n p}(f, g, A) \times(h, l, B)$ if and only if $x \Subset_{n p}$ $(f, g, A)$ and $y \Subset_{n p}(h, l, B)$

(iii) $(x, y) \Subset_{p}(f, g, A) \times(h, l, B)$ if and only if $x \Subset_{p}$ $(f, g, A)$ and $y \Subset_{p}(h, l, B)$

(iv) $(x, y) \Subset_{p t}(f, g, A) \times(h, l, B)$ if and only if $x \Subset_{p t}$ $(f, g, A)$ and $y \Subset_{p t}(h, l, B)$

(v) $(x, y) \Subset_{n t}(f, g, A) \times(h, l, B)$ if and only if $x \Subset_{n t}$ $(f, g, A)$ and $y \Subset_{n t}(h, l, B)$
Proof. We will just prove (iii). The other cases can be proved similarly.

(iii) $(x, y) \Subset_{p}(f, g, A) \times(h, l, B)=(f \times h, g \times l, A \times B)$ $\Leftrightarrow(x, y) \in(f \times h)(a, b)=f(a) \times h(b) \quad$ and $(x, y) \in(g \times l)\left(\neg a^{\prime}, \neg b^{\prime}\right)=g\left(\neg a^{\prime}\right) \times l\left(\neg b^{\prime}\right) \quad$ for some $(a, b) \in A \times B$ and $\left(\neg a^{\prime}, \neg b^{\prime}\right) \in \neg A \times \neg B$

$\Leftrightarrow x \in f(a)$ and $y \in h(b)$ for some $a \in A$ and $b \in B$; $x \in g\left(\neg a^{\prime}\right)$ and $y \in l\left(\neg b^{\prime}\right)$ for some $\neg a^{\prime} \in \neg A$ and $\neg b^{\prime} \in \neg B$

$\Leftrightarrow x \in f(a)$ and $x \in g\left(\neg a^{\prime}\right)$ for some $a \in A$ and $\neg a^{\prime} \in \neg A ; y \in h(b)$ and $y \in l\left(\neg b^{\prime}\right)$ for some $b \in B$ and $\neg b^{\prime} \in \neg B$

$\Leftrightarrow x \Subset_{p}(f, g, A)$ and $y \Subset_{p}(h, l, B)$.

Definition 12. A weak bipolar soft set is a triple $(f, g, A)$ over a nonempty set $X$ with a set of parameters $A$, where $f: A \longrightarrow 2^{X}$ and $g: \neg A \longrightarrow 2^{X}$ are two crisp maps.

A weak bipolar soft set is expressed as a set of ordered triples:

$(f, g, A)=\left\{(a, f(a), g(\neg a)): a \in A\right.$ and $\left.f(a), g(\neg a) \in 2^{X}\right\}$.

A class of all weak bipolar soft sets defined over $X$ with all sets of parameters which are subsets of $A$ is symbolized by $W C\left(X_{A}\right)$.

Remark 2. It is clear that a bipolar soft set is a special case of a weak bipolar soft set, and a weak bipolar soft set is not necessarily a bipolar soft set. We will prove that the image of a bipolar soft set is not necessarily a bipolar soft set; however, the image of a weak bipolar soft set is a weak bipolar soft set. Therefore, we define a soft mapping between two classes of weak bipolar soft sets instead of two classes of bipolar soft sets.

Definition 13. A soft mapping $\pi_{\varphi}$ of $W C\left(X_{A}\right)$ into $W C\left(Y_{B}\right)$ is a pair $(\pi, \varphi)$ of crisp mappings $\pi: X \longrightarrow Y$ and $\varphi: A \longrightarrow B$ such that $\pi_{\varphi}$ is defined as follows: the image of a weak bipolar soft set $\left(f_{1}, f_{2}, M\right)$ in $W C\left(X_{A}\right)$ is a weak bipolar soft set $\pi_{\varphi}\left(f_{1}, f_{2}, M\right)=\left(g_{1}, g_{2}, E\right)$ in $W C\left(Y_{B}\right)$ such that $E=\varphi(M) \subseteq B$ and the two maps $g_{1}$ and $g_{2}$ are given by

$$
g_{1}(e)=\pi\left(\underset{\lambda \in \varphi^{-1}(e) \cap M}{\cup} f_{1}(\lambda)\right), \quad \text { for each } e \in E,
$$

$g_{2}(\neg e)=\pi\left(\underset{\neg \lambda \in \neg\left(\varphi^{-1}(e) \cap M\right)}{\cup} f_{2}(\neg \lambda)\right), \quad$ for each $\neg e \in \neg E$.

The following example shows that the image of a bipolar soft set need not be a bipolar soft set.

Example 5. Consider that $A=\left\{a_{1}, a_{2}, a_{3}\right\}$ and $B=\left\{b_{1}, b_{2}\right\}$ are two sets of parameters and $X=Y=\left\{x_{1}, x_{2}, x_{3}\right\}$ are the universal sets. We define two crisp maps $\pi: X \longrightarrow Y$ and $\varphi: A \longrightarrow B$ as follows: 


$$
\begin{aligned}
& \pi\left(x_{i}\right)=x_{i}, \quad \text { for each } x_{i} \in X, \\
& \varphi\left(a_{1}\right)=b_{1}, \\
& \varphi\left(a_{2}\right)=\varphi\left(a_{3}\right)=b_{2} .
\end{aligned}
$$

Let $M=\left\{a_{2}, a_{3}\right\} \subset A$ and $\left(f_{1}, f_{2}, M\right)$ be a bipolar soft set over $X$ with $M$ defined as follows:

$$
\begin{aligned}
f_{1}\left(a_{2}\right) & =\left\{x_{1}, x_{2}\right\}, \\
f_{1}\left(a_{3}\right) & =\left\{x_{1}\right\}, \\
f_{2}\left(\neg a_{2}\right) & =\left\{x_{3}\right\}, \\
f_{2}\left(\neg a_{3}\right) & =\left\{x_{2}\right\} .
\end{aligned}
$$

Then, $\pi_{\varphi}: W C\left(X_{A}\right) \longrightarrow W C\left(Y_{B}\right)$ is a soft mapping such that the image of $\left(f_{1}, f_{2}, M\right)$ is $\left(g_{1}, g_{2}, E\right)$ in $W C\left(Y_{B}\right)$ such that $E=\varphi(M)=\left\{b_{2}\right\} \subseteq B$ and the two maps $g_{1}$ and $g_{2}$ are given by

$$
\begin{aligned}
g_{1}\left(b_{2}\right) & =\pi\left(f_{1}\left(a_{2}\right) \cup f_{1}\left(a_{3}\right)\right)=\pi\left(\left\{x_{1}, x_{2}\right\}\right)=\left\{x_{1}, x_{2}\right\}, \\
g_{2}\left(\neg b_{2}\right) & =\pi\left(f_{1}\left(\neg a_{2}\right) \cup f\left(\neg a_{3}\right)\right)=\pi\left(\left\{x_{2}, x_{3}\right\}\right)=\left\{x_{2}, x_{3}\right\} .
\end{aligned}
$$

It is clear that $\left(g_{1}, g_{2}, E\right)$ is not a bipolar soft set because $g_{1}\left(b_{2}\right) \cap g_{2}\left(\neg b_{2}\right) \neq \varnothing$.

Proposition 9. Let $\pi_{\varphi}: W C\left(X_{A}\right) \longrightarrow W C\left(Y_{B}\right)$ be a soft mapping such that $\pi: X \longrightarrow Y$ and $\varphi: A \longrightarrow B$ are injective maps. Then, the image of a bipolar soft set is a bipolar soft set.

Proof. Let $\left(f_{1}, f_{2}, M\right)$ be a bipolar soft set in $W C\left(X_{A}\right)$. Then, its image is $\left(g_{1}, g_{2}, E\right)$ in $W C\left(Y_{B}\right)$ such that $E=$ $\varphi(M) \subseteq B$ and the two maps $g_{1}$ and $g_{2}$ are given by

$$
\begin{aligned}
g_{1}(e) & =\pi\left(\bigcup_{\lambda \in \varphi^{-1}(e) \cap M} f_{1}(\lambda)\right), \quad \text { for each } e \in E, \\
g_{2}(\neg e) & =\pi\left(\bigcup_{\neg \lambda \in \neg\left(\varphi^{-1}(e) \cap M\right)} f_{2}(\neg \lambda)\right), \quad \text { for each } \neg e \in \neg E .
\end{aligned}
$$

Since $\varphi$ is injective, $\varphi^{-1}(e)$ is empty or a singleton set. This with the injectiveness of $\pi$ leads to the fact that $g_{1}(e)$ and $g_{2}(\neg e)$ are empty or singleton sets such that $g_{1}(e) \cap g_{2}(\neg e)=\varnothing$ for each $e \in E$. Hence, $\left(g_{1}, g_{2}, E\right)$ is a bipolar soft set.

Definition 14. Let $\pi_{\varphi}: W C\left(X_{A}\right) \longrightarrow W C\left(Y_{B}\right)$ be a soft mapping. Then a crisp map $\varphi^{\prime}: \neg A \longrightarrow \neg B$ is defined as follows: $\varphi^{\prime}(\neg a)=\neg \varphi(a)$, where $\varphi: A \longrightarrow B$ is a crisp map given in Definition 13 .

It is clear that $\varphi^{\prime-1}(\neg b)=\neg \varphi^{-1}(b)$.

Definition 15. A soft map $\pi_{\varphi}: W C\left(X_{A}\right) \longrightarrow W C\left(Y_{B}\right)$ is said to be injective (resp., surjective, bijective) if $\pi_{\varphi}: W C\left(X_{A}\right) \longrightarrow W C\left(Y_{B}\right) a \pi$ and $\varphi$ are injective (resp., surjective, bijective).
Definition 16. Let $\pi_{\varphi}: W C\left(X_{A}\right) \longrightarrow W C\left(Y_{B}\right)$ be a soft mapping. Then, the preimage of a bipolar soft set $\left(h_{1}, h_{2}, N\right)$ in $C\left(Y_{B}\right)$ is a bipolar soft set $\pi_{\varphi}^{-1}\left(h_{1}, h_{2}, N\right)=\left(l_{1}, l_{2}, D\right)$ in $C\left(X_{A}\right)$ such that $D=\varphi^{-1}(N) \subseteq A$ and the two maps $l_{1}$ and $l_{2}$ are given by

$$
\begin{aligned}
l_{1}(d) & =\pi^{-1}\left(h_{1}(\varphi(d)), \quad \text { for each } d \in D,\right. \\
l_{2}(\neg d) & =\pi^{-1}\left(h_{2}(\neg(\varphi(d))), \quad \text { for each } \neg d \in \neg D .\right.
\end{aligned}
$$

Proposition 10. Let $\pi_{\varphi}: W C\left(X_{A}\right) \longrightarrow W C\left(Y_{B}\right)$ be a soft mapping. Then, the preimage of a bipolar soft set is a bipolar soft set.

Proof. Let $\left(h_{1}, h_{2}, N\right)$ be a bipolar soft set in $C\left(Y_{B}\right)$. Then, $\pi_{\varphi}^{-1}\left(h_{1}, h_{2}, N\right)=\left(l_{1}, l_{2}, D\right)$ in $C\left(X_{A}\right)$ such that $D=\varphi^{-1}(N) \subseteq A$ and the two maps $l_{1}$ and $l_{2}$ are given by

$$
\begin{aligned}
l_{1}(d) & =\pi^{-1}\left(h_{1}(\varphi(d))\right), \quad \text { for each } d \in D, \\
l_{2}(\neg d) & =\pi^{-1}\left(h_{2}(\neg(\varphi(d)))\right) \quad \text { for each } \neg d \in \neg D .
\end{aligned}
$$

Let $\varphi(d)=d^{\prime}$.

Then, $\quad l_{1}(d) \cap l_{2}(\neg d)=\pi^{-1}\left(h_{1}\left(d^{\prime}\right) \cap \pi^{-1}\left(h_{2}\left(\neg d^{\prime}\right)\right)=\right.$ $\pi^{-1}\left(h_{1}\left(d^{\prime}\right) \cap h_{2}\left(\neg d^{\prime}\right)\right)=\pi^{-1}(\varnothing)=\varnothing$. Hence, $\left(l_{1}, l_{2}, D\right)$ is a bipolar soft set.

Proposition 11. Let $\pi_{\varphi}: W C\left(X_{A}\right) \longrightarrow W C\left(Y_{B}\right)$ be a soft mapping and let $\left(f_{1}, f_{2}, M\right)$ and $\left(h_{1}, h_{2}, M^{\prime}\right)$ be two bipolar soft sets in $W C\left(X_{A}\right)$. Then, we have the following results:

(i) $\left(\Phi_{\varphi(A)}, X_{\varphi(A)}, \varphi(A)\right) \tilde{\simeq} \pi_{\varphi}\left(\Phi_{A}, X_{A}, A\right)$. The equality holds if $\pi$ and $\varphi$ are surjective.

(ii) $\left(X_{\varphi(A)}, \Phi_{\varphi(A)}, \varphi(A)\right) \tilde{\tilde{s}} \pi_{\varphi}\left(X_{A}, \Phi_{A}, A\right)$. The equality holds if $\pi$ and $\varphi$ are surjective.

(iii) If $\left(f_{1}, f_{2}, M\right) \tilde{\simeq}\left(h_{1}, h_{2}, M^{\prime}\right)$, then $\pi_{\varphi}\left(f_{1}, f_{2}, M\right)$ $\widetilde{\subseteq} \pi_{\varphi}\left(h_{1}, h_{2}, M^{\prime}\right)$.

(iv) $\pi_{\varphi}\left[\left(f_{1}, f_{2}, M\right) \widetilde{U}\left(h_{1}, h_{2}, M^{\prime}\right)\right]=\pi_{\varphi}\left(f_{1}, f_{2}, M\right) \widetilde{U}$ $\pi_{\varphi}\left(h_{1}, h_{2}, M^{\prime}\right)$

(v) $\pi_{\varphi}\left[\left(f_{1}, f_{2}, M\right) \widetilde{\cap}\left(h_{1}, h_{2}, M^{\prime}\right)\right] \widetilde{\simeq} \pi_{\varphi}\left(f_{1}, f_{2}, M\right) \widetilde{\cap} \pi_{\varphi}$ $\left(h_{1}, h_{2}, M^{\prime}\right)$.

The equality holds if $\pi$ and $\varphi$ are injective.

Proof. To prove (i), let $\pi_{\varphi}\left(\Phi_{A}, X_{A}, A\right)=\pi_{\varphi}\left(u_{1}, u_{2}, A\right)=$ $\left(f_{1}, f_{2}, E\right)$, where $u_{1}(a)=\varnothing$ and $E=\varphi(A)$, and $u_{2}(\neg a)=$ $X$ for each $\neg a \in \neg A$. Then, $f_{1}(e)=\pi\left(\cup_{\lambda \in \varphi^{-1}(e)} u_{1}(\lambda)\right)$ $=\pi(\varnothing)=\varnothing \quad$ for each $e \in E$, and $\quad f_{2}(\neg e)=\pi$ $\left(\cup_{\left.\neg \lambda \in \neg \varphi^{-1}(\neg)\right)} u_{2}(\neg \lambda)\right)=\pi(X)=Z \subseteq Y \quad$ for $\quad$ each $\neg e \in \neg E$. Therefore, $\left(f_{1}, f_{2}, E\right)=\left(\Phi_{E}, Z_{E}, E\right)$. Since $E=\varphi(A)$, $f_{1}(e) \subseteq \varnothing \quad$ and $f_{2}(\neg e)=Z \subseteq Y$, then $\left(\Phi_{\varphi(A)}, X_{\varphi(A)}\right.$, $\varphi(A)) \widetilde{\subseteq} \pi_{\varphi}\left(\Phi_{A}, X_{A}, A\right)=\left(\Phi_{E}, Z_{E}, E\right)$.

If $\pi$ and $\varphi$ are surjective, then $E=\varphi(A)=B$ and $\pi(X)=Y$. Hence, $\pi_{\varphi}\left(\Phi_{A}, X_{A}, A\right)=\left(\Phi_{B}, X_{B}, B\right)$.

Following similar argument above, one can prove (ii).

One can prove (iii) easily. 
(iv) First, let $\pi_{\varphi}\left[\left(f_{1}, f_{2}, M\right) \widetilde{U}\left(h_{1}, h_{2}, M^{\prime}\right)\right]=\pi_{\varphi}$ $\left(u_{1}, u_{2}, M \cup M^{\prime}\right)=\left(g_{1}, g_{2}, E\right), \quad$ where $\quad E=\varphi$ $\left(M \cup M^{\prime}\right)$. Now, for each $e \in E$, we have $g_{1}(e)=\pi\left(\cup_{\lambda \in \varphi^{-1}(e) \cap E} u_{1}(\lambda)\right)$. Since

$$
u_{1}(\lambda)= \begin{cases}f_{1}(\lambda), & \lambda \in M-M^{\prime}, \\ h_{1}(\lambda), & \lambda \in M^{\prime}-M, \\ f_{1}(\lambda) \cup h_{1}(\lambda), & \lambda \in M \cap M^{\prime},\end{cases}
$$

then

$$
\begin{aligned}
& \pi\left(\bigcup_{\lambda \in \varphi^{-1}(e) \cap E} u_{1}(\lambda)\right) \\
& \quad=\pi\left(\bigcup \begin{array}{ll}
f_{1}(\lambda) & \lambda \in\left(M-M^{\prime}\right) \cap \varphi^{-1}(e) \\
h_{1}(\lambda) & \lambda \in\left(M^{\prime}-M\right) \cap \varphi^{-1}(e) \\
f_{1}(\lambda) \cup h_{1}(\lambda) & \lambda \in\left(M \cap M^{\prime}\right) \cap \varphi^{-1}(e)
\end{array}\right) .
\end{aligned}
$$

Also, for each $\neg e \in \neg E$, we have $g_{2}(\neg e)=\pi$ $\left(U_{\neg \lambda \in \neg\left(\varphi^{-1}(e) \cap E\right)} \mathcal{u}_{2}(\neg \lambda)\right)$. Since

$$
u_{2}(\neg \lambda)= \begin{cases}f_{2}(\neg \lambda), & \neg \lambda \in \neg M-\neg M^{\prime}, \\ h_{2}(\neg \lambda), & \neg \lambda \in \neg M^{\prime}-\neg M, \\ f_{2}(\neg \lambda) \cup h_{2}(\neg \lambda), & \neg \lambda \in \neg M \cap \neg M^{\prime},\end{cases}
$$

then

$$
\begin{array}{ll}
\pi\left(\bigcup_{\neg \lambda \in \neg\left(\varphi^{-1}(e) \cap E\right)} u_{2}(\neg \lambda)\right) & \\
\quad & \pi\left(\bigcup \begin{array}{ll}
f_{2}(\neg \lambda) & \neg \lambda \in \neg\left[\left(M-M^{\prime}\right) \cap \varphi^{-1}(e)\right] \\
h_{2}(\neg \lambda) & \neg \lambda \in \neg\left[\left(M^{\prime}-M\right) \cap \varphi^{-1}(e)\right] \\
f_{2}(\neg \lambda) \cup h_{2}(\neg \lambda) & \neg \lambda \in \neg\left[\left(M \cap M^{\prime}\right) \cap \varphi^{-1}(e)\right]
\end{array}\right) .
\end{array}
$$

Second, let $\pi_{\varphi}\left(f_{1}, f_{2}, M\right) \widetilde{\cup} \pi_{\varphi}\left(h_{1}, h_{2}, M^{\prime}\right)=\left(w_{1}\right.$, $\left.w_{2}, N\right)$, where $N=\varphi(M) \cup \varphi\left(M^{\prime}\right)$. Now, for each $n \in N$, we have

$$
\begin{aligned}
w_{1}(n) & =\pi\left(\bigcup_{\lambda \in \varphi^{-1}(n) \cap N} f_{1}(\lambda)\right) \cup \pi\left(\bigcup_{\lambda \in \varphi^{-1}(n) \cap N} h_{1}(\lambda)\right) \\
& =\pi\left(\bigcup_{\lambda \in \varphi^{-1}(n) \cap N} f_{1}(\lambda) \cup \bigcup_{\lambda \in \varphi^{-1}(n) \cap N} h_{1}(\lambda)\right) \\
& =\pi\left(\bigcup\left\{\begin{array}{ll}
f_{1}(\lambda) & \lambda \in\left(M-M^{\prime}\right) \cap \varphi^{-1}(n) \\
h_{1}(\lambda) & \lambda \in\left(M^{\prime}-M\right) \cap \varphi^{-1}(n) \\
f_{1}(\lambda) \cup h_{1}(\lambda) & \lambda \in\left(M \cap M^{\prime}\right) \cap \varphi^{-1}(n)
\end{array}\right) .\right.
\end{aligned}
$$

Also, for each $\neg n \in \neg N$, we have

$$
\begin{aligned}
w_{2}(\neg n) & =\pi\left(\bigcup_{\neg \lambda \in \neg\left(\varphi^{-1}(n) \cap N\right)} f_{2}(\neg \lambda)\right) \cup \pi\left(\bigcup_{\neg \lambda \in \neg\left(\varphi^{-1}(n) \cap N\right)} h_{2}(\neg \lambda)\right) \\
& =\pi\left(\bigcup_{\neg \lambda \in \neg\left(\varphi^{-1}(n) \cap N\right)} f_{2}(\neg \lambda) \cup \bigcup_{\neg \lambda \in \neg\left(\varphi^{-1}(n) \cap N\right)} h_{2}(\neg \lambda)\right) \\
& =\pi\left(\begin{array}{ll}
f_{2}(\neg \lambda) & \neg \lambda \in \neg\left[\left(M-M^{\prime}\right) \cap \varphi^{-1}(n)\right] \\
h_{2}(\neg \lambda) & \neg \lambda \in \neg\left[\left(M^{\prime}-M\right) \cap \varphi^{-1}(n)\right] \\
f_{2}(\neg \lambda) \cup h_{2}(\lambda) & \lambda \in \neg\left[\left(M \cap M^{\prime}\right) \cap \varphi^{-1}(n)\right]
\end{array}\right) .
\end{aligned}
$$

Since $\varphi\left(M \cup \varphi M^{\prime}\right)=\varphi(M) \cup \varphi\left(M^{\prime}\right)$, then $E=N$. Thus $v_{1}(e)=w_{1}(e)$ for each $e \in E=N$ and $v_{2}(\neg e)=w_{2}(\neg e)$ for each $\neg e \in \neg E=\neg N$. Hence, we obtain the desired result.

One can prove (v) similarly.

By using a similar technique, one can prove the following result.

Proposition 12. Let $\pi_{\varphi}: W C\left(X_{A}\right) \longrightarrow W C\left(Y_{B}\right)$ be a soft mapping and let $\left(g_{1}, g_{2}, N\right)$ and $\left(l_{1}, l_{2}, N^{\prime}\right)$ be two bipolar soft sets in $W C\left(Y_{B}\right)$. Then, we have the following results:

$$
\begin{aligned}
\text { (i) } \pi_{\varphi}^{-1}\left(\Phi_{B}, X_{B}, B\right) & =\left(\Phi_{A}, X_{A}, A\right) \\
\text { (ii) } \pi_{\varphi}^{-1}\left(X_{B}, \Phi_{B}, B\right) & =\left(X_{A}, \Phi_{A}, A\right)
\end{aligned}
$$

(iii) If $\left(g_{1}, g_{2}, N\right) \tilde{\subseteq}\left(l_{1}, l_{2}, N^{\prime}\right)$, then $\pi_{\varphi}^{-1}\left(g_{1}, g_{2}, N\right)$ $\tilde{\widetilde{c}} \pi_{\varphi}^{-1}\left(l_{1}, l_{2}, N^{\prime}\right)$

(iv) $\pi_{\varphi}^{-1}\left[\left(g_{1}, g_{2}, N\right) \widetilde{U}\left(l_{1}, l_{2}, N^{\prime}\right)\right]=\pi_{\varphi}^{-1}\left(g_{1}, g_{2}, N\right) \widetilde{U}$ $\pi_{\varphi}^{-1}\left(l_{1}, l_{2}, N^{\prime}\right)$

(v) $\pi_{\varphi}^{-1}\left[\left(g_{1}, g_{2}, N\right) \widetilde{\cap}\left(l_{1}, l_{2}, N^{\prime}\right)\right]=\pi_{\varphi}^{-1}\left(g_{1}, g_{2}, N\right) \widetilde{\cap}$ $\pi_{\varphi}^{-1}\left(l_{1}, l_{2}, N^{\prime}\right)$

Proposition 13. Let $\pi_{\varphi}: W C\left(X_{A}\right) \longrightarrow W C\left(Y_{B}\right)$ be a soft mapping and let $\left(f_{1}, f_{2}, M\right)$ be a bipolar soft set in $W C\left(X_{A}\right)$. Then, we have the following results:

(i) If $x \Subset_{p p}\left(f_{1}, f_{2}, M\right)$, then $\pi(x) \Subset_{p p} \pi_{\varphi}\left(f_{1}, f_{2}, M\right)$

(ii) If $x \Subset_{n p}\left(f_{1}, f_{2}, M\right)$, then $\pi(x) \Subset_{n p} \pi_{\varphi}\left(f_{1}, f_{2}, M\right)$ 
(iii) If $x \Subset_{p}\left(f_{1}, f_{2}, M\right)$, then $\pi(x) \Subset_{p} \pi_{\varphi}\left(f_{1}, f_{2}, M\right)$

(iv) If $x \epsilon_{p t}\left(f_{1}, f_{2}, M\right)$, then $\pi(x) \epsilon_{p t} \pi_{\varphi}\left(f_{1}, f_{2}, M\right)$

(v) If $x \epsilon_{n t}\left(f_{1}, f_{2}, M\right)$, then $\pi(x) \epsilon_{n t} \pi_{\varphi}\left(f_{1}, f_{2}, M\right)$

(vi) If $x \notin_{p p}\left(f_{1}, f_{2}, M\right)$ and $\varphi$ is injective, then $\pi(x) \notin_{p p} \pi_{\varphi}\left(f_{1}, f_{2}, M\right)$

(vii) If $x \notin_{n p}\left(f_{1}, f_{2}, M\right)$ and $\varphi$ is injective, then $\pi(x) \notin_{n p} \pi_{\varphi}\left(f_{1}, f_{2}, M\right)$

(viii) If $x \notin_{p}\left(f_{1}, f_{2}, M\right)$ and $\varphi$ is injective, then $\pi(x) \notin_{p} \pi_{\varphi}\left(f_{1}, f_{2}, M\right)$

(ix) If $x \notin_{p t}\left(f_{1}, f_{2}, M\right)$, then $\pi(x) \notin_{p t} \pi_{\varphi}\left(f_{1}, f_{2}, M\right)$

(x) If $x \notin_{n t}\left(f_{1}, f_{2}, M\right)$, then $\pi(x) \notin_{n t} \pi_{\varphi}\left(f_{1}, f_{2}, M\right)$

(xi) If $x \notin{ }_{t}\left(f_{1}, f_{2}, M\right)$, then $\pi(x) \notin{ }_{t} \pi_{\varphi}\left(f_{1}, f_{2}, M\right)$

Proof. We only prove (i), (v), (vi), and (xi). The other cases can be proved similarly.

To prove (i), let $x \Subset_{\mathrm{pp}}\left(f_{1}, f_{2}, M\right)$ and $\pi_{\varphi}\left(f_{1}, f_{2}\right.$, $M)=\left(g_{1}, g_{2}, \varphi(M)\right)$. Then, there exists a parameter $a \in M \subseteq A$ such that $x \in f(a)$. Therefore, there is a parameter $b \in \varphi(M) \subseteq B$ such that $a \in \varphi^{-1}(b)$. Obviously, $a \in \varphi^{-1}$ (b) $\cap M$, so that it follows from Definition 13 that $\pi(x) \in g_{1}(b)=\pi\left(\cup_{\lambda \in \varphi^{-1}(b) \cap M} f_{1}(\lambda)\right) . \quad$ Thus, $\quad \pi(x) \Subset_{\mathrm{pp}}$ $\left(g_{1}, g_{2}, \varphi(M)\right)=\pi_{\varphi}\left(f_{1}, f_{2}, M\right)$, as required.

To prove (v), let $x \Subset_{\mathrm{nt}}\left(f_{1}, f_{2}, M\right)$ and $\pi_{\varphi}\left(f_{1}, f_{2}\right.$, $M)=\left(g_{1}, g_{2}, \varphi(M)\right)$. Then, $x \in f_{2}(\neg a)$ for each $\neg a \in \neg M \subseteq \neg A$. Therefore, for each parameter $\neg b \in \varphi^{\prime}(\neg M)$ $=\neg \varphi(M) \subseteq \neg B$, there is $\neg a \in \varphi^{\prime-1}(\neg b)=\neg \varphi^{-1}(b)$. Obviously, $\neg a \in \neg \varphi^{-1}(b) \cap \neg M$, so that it follows from Definition 13 that for each $\neg b \in \neg \varphi(M)$ we have $\pi(x) \in g_{2}(\neg b)=$ $\pi\left(\cup_{\neg \lambda \in \neg\left(\varphi^{-1}(b) \cap M\right)} f_{2}(\neg \lambda)\right)$. Thus, $\pi(x) \Subset_{\mathrm{nt}}\left(g_{1}, g_{2}, \varphi(M)\right)=$ $\pi_{\varphi}\left(f_{1}, f_{2}, M\right)$, as required.

To prove (vi), let $x \notin_{\mathrm{pp}}(f, g, M)$ and $\pi_{\varphi}\left(f_{1}, f_{2}\right.$, $M)=\left(g_{1}, g_{2}, \varphi(M)\right)$. Then, there exists a parameter $a \in M \subseteq A$ such that $x \notin f_{1}(a)$. Therefore, there is a parameter $b \in \varphi(M) \subseteq B$ such that $a \in \varphi^{-1}(b)$. Since $\varphi$ is injective, $a=\varphi^{-1}(b)$. This means that $\{a\}=\varphi^{-1}(b) \cap M$. Therefore, $\quad \pi(x) \in g_{1}(b)=\pi\left(\cup_{\lambda \in \varphi^{-1}(b) \cap M} f_{1}(\lambda)\right)=\pi$ $\left(f_{1}(a)\right)$. Therefore, $\quad \pi(x) \notin_{\mathrm{pp}}\left(g_{1}, g_{2}, \varphi(M)\right)=\pi_{\varphi}$ $\left(f_{1}, f_{2}, M\right)$, as required.

To prove (xi), let $x \notin{ }_{t}\left(f_{1}, f_{2}, M\right)$ and $\pi_{\varphi}\left(f_{1}, f_{2}, M\right)=\left(g_{1}, g_{2}, \varphi(M)\right)$. Then, $x \notin f_{1}(a)$ for all $a \in M \subseteq A$ and $x \notin f_{2}(\neg a)$ for all $\neg a \in \neg M \subseteq \neg A$. Therefore, for each parameter $b \in \varphi(M) \subseteq B$, there is $a \in M$ such that $a \in \varphi^{-1}(b)$, and for each parameter $\neg b \in \neg \varphi(M) \subseteq \neg B$, there is $\neg a \in \neg M$ such that $\neg a \in \neg \varphi^{-1}(b)$. Thus, we obtain $\pi(x) \notin g_{1}(b)=\pi\left(\cup_{\lambda \in \varphi^{-1}(b) \cap M} f_{1}(\lambda)\right)=\pi\left(f_{1}(a)\right)$ for each $b \in \varphi(M) \quad$ and $\quad \pi(x) \notin g_{2}(\neg b)=\pi\left(\cup_{\neg \lambda \in \neg\left(\varphi^{-1}(b) \cap M\right)}\right.$ $\left.f_{2}(\neg \lambda)\right)=\pi\left(f_{2}(\neg a)\right)$ for each $\neg b \in \neg \varphi(M)$. Hence, $\pi(x) \notin_{t}\left(g_{1}, g_{2}, \varphi(M)\right)=\pi_{\varphi}\left(f_{1}, f_{2}, M\right)$, as required.

Proposition 14. Let $\pi_{\varphi}: W C\left(X_{A}\right) \longrightarrow W C\left(Y_{B}\right)$ be a soft mapping and let $\left(h_{1}, h_{2}, N\right)$ be a bipolar soft set in $W C\left(Y_{B}\right)$. If $\varphi$ is surjective, then we have the following results:

(i) If $y \Subset_{p p}\left(h_{1}, h_{2}, N\right)$, then $x \Subset_{p p} \pi_{\varphi}^{-1}\left(h_{1}, h_{2}, N\right)$ for each $x \in \pi^{-1}(y)$ (ii) If $y \Subset_{n p}\left(h_{1}, h_{2}, N\right)$, then $x \Subset_{n p} \pi_{\varphi}^{-1}\left(h_{1}, h_{2}, N\right)$ for each $x \in \pi^{-1}(y)$

(iii) If $y \Subset_{p}\left(h_{1}, h_{2}, N\right)$, then $x \Subset_{p} \pi_{\varphi}^{-1}\left(h_{1}, h_{2}, N\right)$ for each $x \in \pi^{-1}(y)$

(iv) If $y \epsilon_{p t}\left(h_{1}, h_{2}, N\right)$, then $x \epsilon_{p t} \pi_{\varphi}^{-1}\left(h_{1}, h_{2}, N\right)$ for each $x \in \pi^{-1}(y)$

(v) If $y \epsilon_{n t}\left(h_{1}, h_{2}, N\right)$, then $x \epsilon_{n t} \pi_{\varphi}^{-1}\left(h_{1}, h_{2}, N\right)$ for each $x \in \pi^{-1}(y)$

(vi) If $y \notin_{p p}\left(h_{1}, h_{2}, N\right)$ such that $\pi$ is injective, then $\pi^{-1}(y) \notin_{p p} \pi_{\varphi}^{-1}\left(h_{1}, h_{2}, N\right)$

(vii) If $y \notin_{n p}\left(h_{1}, h_{2}, N\right)$ such that $\pi$ is injective, then $\pi^{-1}(y) \notin_{n p} \pi_{\varphi}^{-1}\left(h_{1}, h_{2}, N\right)$

(viii) If $y \notin_{p}\left(h_{1}, h_{2}, N\right)$ such that $\pi$ is injective, then $\pi^{-1}(y) \notin_{p} \pi_{\varphi}^{-1}\left(h_{1}, h_{2}, N\right)$

(ix) If $y \notin{ }_{p t}\left(h_{1}, h_{2}, N\right)$ such that $\pi$ is injective, then $\pi^{-1}(y) \notin p t \pi_{\varphi}^{-1}\left(h_{1}, h_{2}, N\right)$

(x) If $y \notin{ }_{n t}\left(h_{1}, h_{2}, N\right)$ such that $\pi$ is injective, then $\pi^{-1}(y) \notin_{n t} \pi_{\varphi}^{-1}\left(h_{1}, h_{2}, N\right)$

(xi) If $y \notin{ }_{t}\left(h_{1}, h_{2}, N\right)$ such that $\pi$ is injective, then $\pi^{-1}(y) \notin{ }_{t} \pi_{\varphi}^{-1}\left(h_{1}, h_{2}, N\right)$

Proof. We only prove (i) and (x). The other cases can be proved similarly.

To prove (i), let $y \epsilon_{\mathrm{pp}}\left(h_{1}, h_{2}, N\right)$ and $\pi_{\varphi}^{-1}\left(h_{1}, h_{2}, N\right)=\left(l_{1}, l_{2}, \varphi^{-1}(N)\right)$. Then, there exists a parameter $b \in N \subseteq B$ such that $y \in h(b)$. Since $\varphi$ is surjective, there is a parameter $a \in \varphi^{-1}(N) \subseteq A$ such that $\varphi(a)=b$. It follows from Definition 16 that $l_{1}(a)=\pi^{-1}$ $\left(h_{1}(\varphi(a))=\pi^{-1}\left(h_{1}(b)\right)\right.$. Now, for each $x \in \pi^{-1}(y)$, we obtain $x \Subset_{\mathrm{pp}}\left(l_{1}, l_{2}, \varphi^{-1}(N)\right)=\pi_{\varphi}^{-1}\left(h_{1}, h_{2}, N\right)$, as required.

To prove (x), let $y \notin_{\mathrm{nt}}\left(h_{1}, h_{2}, N\right)$ and $\pi_{\varphi}^{-1}\left(h_{1}, h_{2}, N\right)=\left(l_{1}, l_{2}, \varphi^{-1}(N)\right)$. Then, $y \notin h_{2}(\neg b)$ for all $\neg b \in \neg N \subseteq \neg B$. Since $\varphi$ is surjective, there exists a parameter $\neg a \in \neg \varphi^{-1}(N) \subseteq \neg A$ such that $\neg \varphi(a)=\neg b$. It follows from Definition 16 that $l_{2}(\neg a)=\pi^{-1} \quad\left(h_{2}(\neg \varphi(a))=\pi^{-1}\right.$ $\left(h_{2}(\neg b)\right)$. Since $\pi$ is injective, $\pi^{-1}(y) \notin_{\mathrm{nt}}\left(l_{1}, l_{2}, \varphi^{-1}\right.$ $(N))=\pi_{\varphi}^{-1}\left(h_{1}, h_{2}, N\right)$, as required.

\section{Application of Bipolar Soft Sets}

In this section, we apply the idea of bipolar soft sets to initiate an application of optimal choices. We provide an example to demonstrate how we make optimal choices. Then, we construct an algorithm of this method.

Example 6. Suppose that a car sales company has a set of cars $X$ with a set of parameters $E$. Let $X=\left\{c_{i}: i=1,2, \ldots, 20\right\}$ be a set of twenty cars and $A=$ $\left\{a_{i}: i=1,2, \ldots, 11\right\}$ be a set of eleven parameters, where $a_{i}(i=1,2,3,4,5,6,7,8,9,10,11)$ stand for "expensive," "cheap," "modern," "sport," "red color," "white color," "Japanese industry," "German industry," "in good repair," "in bad repair," and "low fuel consumption," respectively.

It should be noted that $\neg a_{1}$ does not mean "cheap" and $\neg a_{2}$ does not mean "expensive." 
Now, suppose that a car sales company classifies these cars with respect to the set of parameters using a notion of a bipolar soft set $(f, g, A)$ as follows:

$$
\begin{aligned}
& f\left(a_{1}\right)=\left\{c_{i}: i=4,7,8, \ldots, 12\right\}, g\left(\neg a_{1}\right)=\left\{c_{i}: i=14,15, \ldots, 18\right\}, \\
& f\left(a_{2}\right)=\left\{c_{i}: i=5,10,14,15,18\right\}, g\left(\neg a_{2}\right)=\left\{c_{i}: i=7,8,9,11,12,19\right\}, \\
& f\left(a_{3}\right)=\left\{c_{i}: i=1,2,3,6,8,10,19,20\right\}, g\left(\neg a_{3}\right)=\left\{c_{i}: i=9,13,16,17\right\}, \\
& f\left(a_{4}\right)=\left\{c_{i}: i=4,9,15,16,17\right\}, g\left(\neg a_{4}\right)=\left\{c_{i}: i=8,10,11,12,13,20\right\}, \\
& f\left(a_{5}\right)=\left\{c_{i}: i=1,2, \ldots, 7\right\}, g\left(\neg a_{5}\right)=\left\{c_{i}: i=8,9, \ldots, 20\right\}, \\
& f\left(a_{6}\right)=\left\{c_{i}: i=1,4,8,12,13, \ldots, 16,20\right\}, g\left(\neg a_{6}\right)=\left\{c_{i}: i=2, \ldots, 7,9,10,11,17,18,19\right\}, \\
& f\left(a_{7}\right)=\left\{c_{i}: i=1,2, \ldots, 7,14,16\right\}, g\left(\neg a_{7}\right)=\left\{c_{i}: i=8,9, \ldots, 13,15,17,18,19,20\right\}, \\
& f\left(a_{8}\right)=\left\{c_{i}: i=4,8,10,13,15,18,19,20\right\}, g\left(\neg a_{8}\right)=\left\{c_{i}: i=1,2,3,5,6,7,9,11,12,14,16,17\right\}, \\
& f\left(a_{9}\right)=\left\{c_{i}: i=1,4,5,8,15,16, \ldots, 20\right\}, g\left(\neg a_{9}\right)=\left\{c_{i}: i=9,11,12,14\right\}, \\
& f\left(a_{10}\right)=\left\{c_{i}: i=9,11,12\right\}, g\left(\neg a_{10}\right)=\left\{c_{i}: i=1,5,8,15,16, \ldots, 20\right\}, \\
& f\left(a_{11}\right)=\left\{c_{i}: i=1,3,5,8,11, \ldots, 17\right\}, g\left(\neg a_{11}\right)=\left\{c_{i}: i=4,6,7,18\right\} .
\end{aligned}
$$

Now, suppose that Mr. Redhwan wants to choose a car with respect to a set of parameters $E=\left\{a_{i}: i=2,3,6,8,9,11\right\} \subseteq A$. To help him, we will construct two tables, one of them with respect to a map $f: E \longrightarrow 2^{X}$ (see Table 1) and the other with respect to a map $g: \neg E \longrightarrow 2^{X}$ (see Table 2). Then we determine the value of $\left(c_{k}, f\left(a_{i}\right)\right)$ and $\left(c_{k}, g\left(\neg a_{i}\right)\right)$ by the following two roles:

$$
\begin{gathered}
\left(c_{k}, f\left(a_{i}\right)\right)= \begin{cases}1, & c_{k} \in f\left(a_{i}\right), \\
0, & c_{k} \notin f\left(a_{i}\right),\end{cases} \\
\left(c_{k}, g\left(\neg a_{i}\right)\right)= \begin{cases}-1, & c_{k} \in g\left(\neg a_{i}\right), \\
0, & c_{k} \notin g\left(\neg a_{i}\right) .\end{cases}
\end{gathered}
$$

Since $f\left(a_{i}\right) \cap g\left(\neg a_{i}\right)=\varnothing$ for each $a_{i} \in E$, we can combine Tables 1 and 2 in Table 3. Note that the value of $\left(c_{k},\left(f\left(a_{i}\right), g\left(\neg a_{i}\right)\right)\right)$ is given by the following two roles:

$$
\left(c_{k},\left(f\left(a_{i}\right), g\left(\neg a_{i}\right)\right)\right)= \begin{cases}1, & c_{k} \in f\left(a_{i}\right), \\ -1, & c_{k} \in g\left(\neg a_{i}\right), \\ 0, & c_{k} \notin f\left(a_{i}\right) \cup g\left(\neg a_{i}\right) .\end{cases}
$$

One can note from Table 3 that car no. 15 is the optimal car for Mr. Redhwan. Cars no. 8 and no. 20 are the second optimal cars for Mr. Redhwan.

In the following, we present an algorithm for determining the wining students.

Step 1. Determine a set of parameters $A$ and the universal set $X$.

Step 2. Define a map $f$ which associates each parameter of $A$ with its corresponding subset of $2^{X}$.

Step 3. Define a map $g$ which associates each parameter of $\neg A$ with its corresponding subset of $2^{X}$.
Step 4. Determine the favorite set of parameters $E \subseteq A$ of Mr. X.

Step 5. Construct a bipolar soft set $(f, g, E)$.

Step 6. Initiate a table which represents a bipolar soft set $(f, g, E)$ (see Table 3 ).

Step 7. Input the value of $\left(c_{k},\left(f\left(a_{i}\right), g\left(\neg a_{i}\right)\right)\right)$ as given by the following two roles:

$$
\left(c_{k},\left(f\left(a_{i}\right), g\left(\neg a_{i}\right)\right)\right)= \begin{cases}1, & c_{k} \in\left(f\left(a_{i}\right)\right), \\ -1, & c_{k} \in g\left(\neg a_{i}\right), \\ 0, & c_{k} \notin f\left(a_{i}\right) \cup g\left(\neg a_{i}\right) .\end{cases}
$$

Step 8. Count the values of each arrow by the rule $\operatorname{Sum}_{k}=\sum_{i=1}^{m}\left(c_{k},\left(f\left(a_{i}\right), g\left(\neg a_{i}\right)\right)\right)$.

Step 9. Find the decision, denoted by $d$, for which $S=\max \left\{\operatorname{Sum}_{k}: k=1,2, \ldots, s\right\}$, where $s=|X|$.

Step 10. Then, $d$ is the optimal choice car. If $d$ has more than one value, then any one of them could be chosen by Mr. Redhwan satisfying his option.

On the other hand, some of the parameters are of less significance than the other ones so they must be graded with lesser priority. For this reason, we suggest weights $w_{i} \in(0,1]$ on the parameters according to the desire of customers.

In this case, we modify the previous algorithm to be convenient for weighted selection.

Step 1. Determine a set of parameters $A$ and the universal set $X$.

Step 2. Define a map $f$ which associates each parameter of $A$ with its corresponding subset of $2^{X}$. 
TABLE 1: The positive components $f$ of bipolar soft sets $(f, g, E)$ of each car.

\begin{tabular}{|c|c|c|c|c|c|c|}
\hline \multirow{2}{*}{ Cars } & \multicolumn{6}{|c|}{$f\left(a_{i}\right)$} \\
\hline & $a_{2}$ & $a_{3}$ & $a_{6}$ & $a_{8}$ & $a_{9}$ & $a_{11}$ \\
\hline$c_{1}$ & 0 & 1 & 1 & 0 & 1 & 1 \\
\hline$c_{2}$ & 0 & 1 & 0 & 0 & 0 & 0 \\
\hline$c_{3}$ & 0 & 1 & 0 & 0 & 0 & 1 \\
\hline$c_{4}$ & 1 & 0 & 1 & 1 & 1 & 0 \\
\hline$c_{5}$ & 1 & 0 & 0 & 0 & 1 & 1 \\
\hline$c_{6}$ & 0 & 1 & 0 & 0 & 0 & 0 \\
\hline$c_{7}$ & 0 & 0 & 0 & 0 & 0 & 0 \\
\hline$c_{8}$ & 0 & 1 & 1 & 1 & 1 & 1 \\
\hline$c_{9}$ & 0 & 0 & 0 & 0 & 0 & 0 \\
\hline$c_{10}$ & 1 & 1 & 0 & 1 & 0 & 0 \\
\hline$c_{11}$ & 0 & 0 & 0 & 0 & 0 & 1 \\
\hline$c_{12}$ & 0 & 0 & 1 & 0 & 0 & 1 \\
\hline$c_{13}$ & 0 & 0 & 1 & 1 & 0 & 1 \\
\hline$c_{14}$ & 1 & 0 & 1 & 0 & 0 & 1 \\
\hline$c_{15}$ & 1 & 0 & 1 & 1 & 1 & 1 \\
\hline$c_{16}$ & 0 & 0 & 1 & 0 & 1 & 1 \\
\hline$c_{17}$ & 0 & 0 & 0 & 0 & 1 & 1 \\
\hline$c_{18}$ & 1 & 0 & 0 & 1 & 1 & 0 \\
\hline$c_{19}$ & 0 & 1 & 0 & 1 & 1 & 0 \\
\hline$c_{20}$ & 0 & 1 & 1 & 1 & 1 & 0 \\
\hline
\end{tabular}

TABLE 2: The negative components $g$ of bipolar soft sets $(f, g, E)$ of each car.

\begin{tabular}{|c|c|c|c|c|c|c|}
\hline \multirow{2}{*}{ Cars } & \multicolumn{6}{|c|}{$g\left(\neg a_{i}\right)$} \\
\hline & $\neg a_{2}$ & $\neg a_{3}$ & $\neg a_{6}$ & $\neg a_{8}$ & $\neg a_{9}$ & $\neg a_{11}$ \\
\hline$c_{1}$ & 0 & 0 & 0 & -1 & 0 & 0 \\
\hline$c_{2}$ & 0 & 0 & -1 & -1 & 0 & 0 \\
\hline$c_{3}$ & 0 & 0 & -1 & -1 & 0 & 0 \\
\hline$c_{4}$ & 0 & 0 & 0 & 0 & 0 & -1 \\
\hline$c_{5}$ & 0 & 0 & -1 & -1 & 0 & 0 \\
\hline$c_{6}$ & 0 & 0 & -1 & -1 & 0 & -1 \\
\hline$c_{7}$ & -1 & 0 & -1 & -1 & 0 & -1 \\
\hline$c_{8}$ & -1 & 0 & 0 & 0 & 0 & 0 \\
\hline$c_{9}$ & -1 & -1 & -1 & -1 & -1 & 0 \\
\hline$c_{10}$ & 0 & 0 & -1 & 0 & 0 & 0 \\
\hline$c_{11}$ & -1 & 0 & -1 & -1 & -1 & 0 \\
\hline$c_{12}$ & -1 & 0 & 0 & -1 & -1 & 0 \\
\hline$c_{13}$ & 0 & -1 & 0 & 0 & 0 & 0 \\
\hline$c_{14}$ & 0 & 0 & 0 & -1 & -1 & 0 \\
\hline$c_{15}$ & 0 & 0 & 0 & 0 & 0 & 0 \\
\hline$c_{16}$ & 0 & -1 & 0 & -1 & 0 & 0 \\
\hline$c_{17}$ & 0 & -1 & -1 & -1 & 0 & 0 \\
\hline$c_{18}$ & 0 & 0 & -1 & 0 & 0 & -1 \\
\hline$c_{19}^{10}$ & -1 & 0 & -1 & 0 & 0 & 0 \\
\hline$c_{20}$ & 0 & 0 & 0 & 0 & 0 & 0 \\
\hline
\end{tabular}

Step 3. Define a map $g$ which associates each parameter of $\neg A$ with its corresponding subset of $2^{X}$.

Step 4. Determine the favorite set of parameters $E \subseteq A$ of Mr. X.

Step 5. Determine the weight $w_{a}$ of each parameter of the favorite set of parameters.

Step 6. Construct a bipolar soft set $(f, g, E)$.

Step 7. Initiate a table which represents a bipolar soft set $(f, g, E)$ (see Table 3 ).
Step 8. Input the value of $\left(c_{k},\left(f\left(a_{i}\right), g\left(\neg a_{i}\right)\right)\right)$ as given by the following two roles:

$$
\left(c_{k},\left(f\left(a_{i}\right), g\left(\neg a_{i}\right)\right)\right)= \begin{cases}w_{a_{i}}, & c_{k} \in\left(f\left(a_{i}\right),\right. \\ -w_{a_{i}}, & c_{k} \in g\left(\neg a_{i}\right), \\ 0, & c_{k} \notin f\left(a_{i}\right) \cup g\left(\neg a_{i}\right) .\end{cases}
$$

Step 9. Count the values of each arrow by the rule $\operatorname{Sum}_{k}=\sum_{i=1}^{m}\left(c_{k},\left(f\left(a_{i}\right), g\left(\neg a_{i}\right)\right)\right)$. See Table 4 . 
TABLE 3: Values of all components of the favorite bipolar soft sets $(f, g, E)$.

\begin{tabular}{|c|c|c|c|c|c|c|c|}
\hline \multirow{2}{*}{ Cars } & \multicolumn{7}{|c|}{$(f(a), g(\neg a))$} \\
\hline & $\left(a_{2}, \neg a_{2}\right)$ & $\left(a_{3}, \neg a_{3}\right)$ & $\left(a_{6}, \neg a_{6}\right)$ & $\left(a_{8}, \neg a_{8}\right)$ & $\left(a_{9}, \neg a_{9}\right)$ & $\left(a_{11}, \neg a_{11}\right)$ & Sum \\
\hline$c_{1}$ & 0 & 1 & 1 & -1 & 1 & 1 & 3 \\
\hline$c_{2}$ & 0 & 1 & -1 & -1 & 0 & 0 & -1 \\
\hline$c_{3}$ & 0 & 1 & -1 & -1 & 0 & 1 & 0 \\
\hline$c_{4}$ & 1 & 0 & 1 & 1 & 1 & -1 & 3 \\
\hline$c_{5}$ & 1 & 0 & -1 & -1 & 1 & 1 & 1 \\
\hline$c_{6}$ & 0 & 1 & -1 & -1 & 0 & -1 & -2 \\
\hline$c_{7}$ & -1 & 0 & -1 & -1 & 0 & -1 & -4 \\
\hline$c_{8}$ & -1 & 1 & 1 & 1 & 1 & 1 & 4 \\
\hline$c_{9}$ & -1 & -1 & -1 & -1 & -1 & 0 & -5 \\
\hline$c_{10}$ & 1 & 1 & -1 & 1 & 0 & 0 & 2 \\
\hline$c_{11}$ & -1 & 0 & -1 & -1 & -1 & 1 & -3 \\
\hline$c_{12}$ & -1 & 0 & 1 & -1 & -1 & 1 & -1 \\
\hline$c_{13}$ & 0 & -1 & 1 & 1 & 0 & 1 & 2 \\
\hline$c_{14}$ & 1 & 0 & 1 & -1 & -1 & 1 & 1 \\
\hline$c_{15}$ & 1 & 0 & 1 & 1 & 1 & 1 & 5 \\
\hline$c_{16}$ & 0 & -1 & 1 & -1 & 1 & 1 & 1 \\
\hline$c_{17}$ & 0 & -1 & -1 & -1 & 1 & 1 & -1 \\
\hline$c_{18}$ & 1 & 0 & -1 & 1 & 1 & -1 & 1 \\
\hline$c_{19}$ & -1 & 1 & -1 & 1 & 1 & 0 & 1 \\
\hline$c_{20}$ & 0 & 1 & 1 & 1 & 1 & 0 & 4 \\
\hline
\end{tabular}

TABLE 4: Weight values of all components of the favorite bipolar soft sets $(f, g, E)$.

\begin{tabular}{|c|c|c|c|c|c|c|c|}
\hline Cars & $\left(a_{2}, \neg a_{2}\right), 10 \%$ & $\left(a_{3}, \neg a_{3}\right), 40 \%$ & $\left(a_{6}, \neg a_{6}\right), 10 \%$ & $\begin{array}{l}(f, g), w_{a_{i}} \\
\quad\left(a_{8}, \neg a_{8}\right), 10 \%\end{array}$ & $\left(a_{9}, \neg a_{9}\right), 20 \%$ & $\left(a_{11}, \neg a_{11}\right), 10 \%$ & Sum \\
\hline$c_{1}$ & 0 & 0.4 & 0.1 & -0.1 & 0.2 & 0.1 & 0.7 \\
\hline$c_{2}$ & 0 & 0.4 & -0.1 & -0.1 & 0 & 0 & -0.2 \\
\hline$c_{3}$ & 0 & 0.4 & -0.1 & -0.1 & 0 & 0.1 & 0.3 \\
\hline$c_{4}$ & 0.1 & 0 & 0.1 & 0.1 & 0.2 & -0.1 & 0.4 \\
\hline$c_{5}$ & 0.1 & 0 & -0.1 & -0.1 & 0.2 & 0.1 & 0.2 \\
\hline$c_{6}$ & 0 & 0.4 & -0.1 & -0.1 & 0 & -0.1 & 0.1 \\
\hline$c_{7}$ & -0.1 & 0 & -0.1 & -0.1 & 0 & -0.1 & -0.4 \\
\hline$c_{8}$ & -0.1 & 0.4 & 0.1 & 0.1 & 0.2 & 0.1 & 0.8 \\
\hline$c_{9}$ & -0.1 & -0.4 & -0.1 & -0.1 & -0.2 & 0 & -0.9 \\
\hline$c_{10}$ & 0.1 & 0.4 & -0.1 & 0.1 & 0 & 0 & 0.5 \\
\hline$c_{11}$ & -0.1 & 0 & -0.1 & -0.1 & -0.2 & 0.1 & -0.4 \\
\hline$c_{12}$ & -0.1 & 0 & 0.1 & -0.1 & -0.2 & 0.1 & -0.2 \\
\hline$c_{13}^{12}$ & 0 & -0.4 & 0.1 & 0.1 & 0 & 0.1 & -0.1 \\
\hline$c_{14}$ & 0.1 & 0 & 0.1 & -0.1 & -0.2 & 0.1 & -0.2 \\
\hline$c_{15}$ & 0.1 & 0 & 0.1 & 0.1 & 0.2 & 0.1 & 0.6 \\
\hline$c_{16}$ & 0 & -0.4 & 0.1 & -0.1 & 0.2 & 0.1 & -0.1 \\
\hline$c_{17}$ & 0 & -0.4 & -0.1 & -0.1 & 0.2 & 0.1 & -0.3 \\
\hline$c_{18}$ & 0.1 & 0 & -0.1 & 0.1 & 0.2 & -0.1 & 0.2 \\
\hline$c_{19}$ & -0.1 & 0.4 & -0.1 & 0.1 & 0.2 & 0 & 0.5 \\
\hline$c_{20}$ & 0 & 0.4 & 0.1 & 0.1 & 0.2 & 0 & 0.8 \\
\hline
\end{tabular}

Step 10. Find the decision, denoted by $d$, for which $S=\max \left\{\operatorname{Sum}_{k}: k=1,2, \ldots, s\right\}$, where $s=|X|$.

Step 11. Then, $d$ is the optimal choice car. If $d$ has more than one value, then any one of them could be chosen by Mr. Redhwan satisfying his option.

With respect to our example, suppose that the weights $10 \%, 40 \%, 10 \%, 10 \%, 20 \%$, and $10 \%$ are, respectively, corresponding to $a_{2}, a_{3}, a_{6}, a_{8}, a_{9}$, and $a_{11}$. Then, we update Table 3 to be as shown in Table 4 .
Now, one can note from Table 4 that cars no. 8 and no. 20 are the optimal cars for Mr. Redhwan. Therefore, any one of them could be chosen by Mr. Redhwan satisfying his option.

\section{Conclusion}

This study has introduced five types of belong relations and six types of nonbelong relations between a bipolar soft set and an ordinary point. These relations can be considered as a primary indicator of membership and nonmembership 
degree of an element. Then, the concept of soft mappings between two classes of bipolar soft sets has been defined, and the sufficient conditions to preserve these relations between an ordinary point and its image and preimage have been studied. Finally, we have applied the idea of bipolar soft sets to present an application of choosing the best products according to the favorite set of parameters. We have given an algorithm of the application and provided explanatory example.

In the upcoming work, we shall exploit these relations to initiate different types of soft separation axioms and compact spaces on bipolar soft topological spaces. In addition, we shall try to model some natural phenomena using bipolar soft sets.

\section{Data Availability}

No data were used to support this study.

\section{Conflicts of Interest}

The author declares that he has no conflicts of interest.

\section{References}

[1] L. A. Zadeh, "Fuzzy sets," Information and Control, vol. 8, no. 3, pp. 338-353, 1965.

[2] K. T. Atanassov, "Intuitionistic fuzzy sets," Fuzzy Sets and Systems, vol. 20, no. 1, pp. 87-96, 1986.

[3] Z. A. Pawlak, "Rough sets," International Journal of Computer \& Information Sciences, vol. 11, no. 5, p. 341, 1982.

[4] D. Molodtsov, "Soft set theory-first results," Computers \& Mathematics with Applications, vol. 37, no. 4-5, pp. 19-31, 1999.

[5] M. I. Ali, F. Feng, X. Liu, W. K. Min, and M. Shabir, "On some new operations in soft set theory," Computers \& Mathematics with Applications, vol. 57, no. 9, pp. 1547-1553, 2009.

[6] T. M. Al-shami, "Comment on "soft mappings spaces"” The Scientific World Journal, vol. 2019, Article ID 6903809, 2 pages, 2019.

[7] T. M. Al-shami, "Investigation and corrigendum to some results related to g-soft equality and $g f$-soft equality relations," Filomat, vol. 33, no. 11, pp. 3375-3383, 2019.

[8] T. M. Al-shami, "Comments on some results related to soft separation axioms," Afrika Matematika, vol. 31, no. 7-8, pp. 1105-1119, 2020.

[9] T. M. Al-shami and M. E. El-Shafei, "T-soft equality relation," Turkish Journal of Mathematics, vol. 44, no. 4, pp. 1427-1441, 2020.

[10] N. Cağman and S. Enginoğlu, "Soft matrix theory and its decision making," Computers and Mathematics with Applications, vol. 59, no. 10, pp. 3308-3314, 2010.

[11] D. Chen, E. C. C. Tsang, D. S. Yeung, and X. Wang, "The parameterization reduction of soft sets and its applications," Computers \& Mathematics with Applications, vol. 49, no. 5-6, pp. 757-763, 2005.

[12] P. K. Maji, R. Biwas, and A. R. Roy, "Fuzzy soft sets," Journal of Fuzzy Mathematics, vol. 9, pp. 589-602, 2001.

[13] P. K. Maji, A. R. Biswas, and R. Roy, "An application of soft sets in a decision making problem," Computers \& Mathematics with Applications, vol. 44, no. 8-9, pp. 1077-1083, 2002.
[14] F. Feng, C. Li, B. Davvaz, and M. I. Ali, "Soft sets combined with fuzzy sets and rough sets: a tentative approach," Soft Computing, vol. 14, no. 9, pp. 899-911, 2010.

[15] F. Feng, X. Liu, V. Leoreanu-Fotea, and Y. B. Jun, "Soft sets and soft rough sets," Information Sciences, vol. 181, no. 6, pp. 1125-1137, 2011.

[16] Y. B. Jun and S. S. Ahn, "Double-framed soft sets with applications in BCK/BCI-algebras," Journal of Applied Mathematics, vol. 2012, Article ID 178159, 15 pages, 2012.

[17] M. Shabir and M. Naz, "On bipolar soft sets," 2013, http:// arxiv.org/abs/1303.1344v1.

[18] A. Ackgoz and N. Tas, "Binary soft set theory," European Journal of Pure and Applied Mathematics, vol. 9, no. 4, pp. 452-463, 2016.

[19] F. Fatimah, D. Rosadi, R. B. F. Hakim, and J. C. R. Alcantud, "N-soft sets and their decision making algorithms," Soft Computing, vol. 22, no. 12, pp. 3829-3842, 2018.

[20] M. Naz and M. Shabir, "On fuzzy bipolar soft sets, their algebraic structures and applications," Journal of Intelligent \& Fuzzy Systems, vol. 26, no. 4, pp. 1645-1656, 2014.

[21] S. Abdullah, M. Aslam, and K. Ullah, "Bipolar fuzzy soft sets and its applications in decision making problem," Journal of Intelligent \& Fuzzy Systems, vol. 27, no. 2, pp. 729-742, 2014.

[22] F. Karaaslan and N. Çağman, "Bipolar soft rough sets and their applications in decision making," Afrika Matematika, vol. 29, no. 5-6, pp. 823-839, 2018.

[23] M. Shabir and R. Gul, "Modified rough bipolar soft sets," Journal of Intelligent \& Fuzzy Systems, vol. 39, no. 3, Article ID 4259, 2020.

[24] D. Dubois and H. Prade, "An introduction to bipolar representations of information and preference," International Journal of Intelligent Systems, vol. 23, no. 8, pp. 866-877, 2008.

[25] F. Karaaslan and S. Karataş, "A new approach to bipolar soft sets and its applications," Discrete Mathematics, Algorithms and Applications, vol. 7, no. 4, 14 pages, Article ID 1550054, 2015.

[26] K. Hayat and T. Mahmood, "Some applications of bipolar soft set: characterizations of two isomorphic hemi-rings via BSI-hideals," British Journal of Mathematics \& Computer Science, vol. 13, no. 2, pp. 1-21, 2016.

[27] F. Karaaslan, I. Ahmad, and A. Ullah, "Bipolar soft groups," Journal of Intelligent \& Fuzzy Systems, vol. 31, no. 1, pp. 651-662, 2016.

[28] M. Shabir and A. Bakhtawar, "Bipolar soft connected, bipolar soft disconnected and bipolar soft compact spaces," Songklanakarin Journal of Science and Technology, vol. 39, no. 3, Article ID 359371, 2017.

[29] T. Y. Öztürk, "On bipolar soft topological spaces," Journal of New Theory, vol. 20, pp. 64-75, 2018.

[30] A. Fadel and N. Hassan, "Separation axioms of bipolar soft topological space," Journal of Physics: Conference Series, vol. 1212, Article ID 012017, 2019.

[31] A. Fadel and S. C. Dzul-Kifli, "Bipolar soft topological spaces," European Journal of Pure and Applied Mathematics, vol. 13, no. 2, pp. 227-245, 2020.

[32] M. E. El-Shafei, M. Abo-Elhamayel, and T. M. Al-shami, "Partial soft separation axioms and soft compact spaces," Filomat, vol. 32, no. 13, pp. 4755-4771, 2018.

[33] T. M. Al-shami, "Soft separation axioms and fixed soft points using soft semiopen sets," Journal of Applied Mathematics, vol. 2020, Article ID 1746103, 11 pages, 2020.

[34] T. M. Al-shami and M. E. El-Shafei, "Partial belong relation on soft separation axioms and decision-making problem, two 
birds with one stone," Soft Computing, vol. 24, no. 7, pp. 5377-5387, 2020.

[35] M. E. El-Shafei and T. M. Al-shami, "Applications of partial belong and total non-belong relations on soft separation axioms and decision-making problem," Computational and Applied Mathematics, vol. 39, Article ID 138, 2020.

[36] T. M. Al-shami and M. E. El-Shafei, "On supra soft topological ordered spaces," Arab Journal of Basic and Applied Sciences, vol. 26, no. 1, pp. 433-445, 2019.

[37] T. M. Al-shami and M. E. El-Shafei, "Two types of separation axioms on supra soft topological spaces," Demonstratio Mathematica, vol. 52, no. 1, pp. 147-165, 2019.

[38] T. M. Al-shami, M. E. El-Shafei, and M. Abo-Elhamayel, "On soft topological ordered spaces," Journal of King Saud University-Science, vol. 31, no. 4, pp. 556-566, 2019. 